\title{
Estimation du niveau de dégradation d'un processus à l'aide de lois de dégradation et d'un modèle de Markov caché
}

\author{
Pascal Vrignat* - Manuel Avila* - Florent Duculty* - Sébastien \\ Aupetit** - Mohamed Slimane** - Frédéric Kratz*** \\ * Université d'Orléans - Laboratoire PRISME - Equipe MCDS - \\ IUT de l'Indre - 2 avenue François Mitterrand \\ 36000 Châteauroux \\ \{pascal.vrignat, manuel.avila,florent.duculty\}@univ-orleans.fr \\ ** Université François Rabelais Tours - Laboratoire d'Informatique \\ Polytech'Tours - 64 avenue Jean Portalis \\ 37200 Tours \\ \{sebastien.aupetit, mohamed.slimane\}@univ-tours.fr \\ *** ENSI de Bourges - Laboratoire PRISME - Equipe MCDS \\ ENSI - 88 boulevard Lahitolle \\ 18020 Bourges Cedex \\ frederic.kratz@ensi-bourges.fr
}

\begin{abstract}
RÉSUMÉ. De nos jours, les stratégies de maintenance et leurs évaluations demeurent une préoccupation particulièrement forte au sein des entreprises. Une suite d'évènements particuliers peut, éventuellement, informer l'expert d'une panne prochaine. Notre étude tente d'appréhender "cette signature " à l'aide de modèles de Markov cachés. Nous proposons à l'expert deux méthodes sur l'estimation du niveau de dégradation du système maintenu. La première utilise des lois de dégradation non paramétrique et semi- paramétrique qui serviront de référence. La deuxième consiste à utiliser une approche markovienne.

ABSTRACT. Today, maintenance strategies and their analyses remain a worrying problem for companies. A series of specific events can potentially warn the expert of an imminent breakdown. Our study aims at understanding this "signature" thanks to hidden Markov model. On that purpose, two methods on damage level estimation of a maintained system are proposed to experts. The first one consists in using non parametric and semiparametric degradation laws (They serve as a reference). The second one consists in using a markovian approach.

MOTS-CLÉS : diagnostic, gestion de maintenance, évaluation, lois de dégradation, modèle de Markov caché.

KEYWORDS: diagnosis, maintenance management, assessment, degradation laws, Hidden Markov Model.
\end{abstract}

Revue. Volume $\mathrm{X}-\mathrm{n}^{\circ} \mathrm{x} /$ année, pages 1 à $\mathrm{X}$ 


\section{Introduction}

Dans l'entreprise, la fonction "maintenance " consiste de moins en moins souvent à remettre en état l'outil de production mais de plus en plus fréquemment à anticiper ses dysfonctionnements. L'arrêt ou le fonctionnement anormal de celui-ci, et le non-respect des délais qui s'en suivent, engendrent des coûts que l'entreprise n'est plus capable de supporter. Elle ne peut plus attendre que la panne se produise pour y remédier, mais doit désormais s'organiser pour procéder aux diverses opérations qui permettent de l'éviter. Nous sommes ainsi passés d'une «maintenance curative » à une " maintenance préventive ». Ce phénomène se traduit par la définition de plans d'actions et d'interventions sur le système ou processus. Cela permet de remplacer des composants juste avant leur défaillance. Ces actions préventives étaient dans un premier temps effectuées de façon systématique selon des calendriers prédéfinis. Elles permettaient effectivement d'anticiper les pannes, mais au prix d'un alourdissement important des coûts de maintenance. "Une nouvelle maintenance " se développe aujourd'hui grâce aux différents travaux de recherche et aux technologies de diagnostic et de contrôle utilisant les réseaux de communication (Beltrami, 2005; Leger, 2007; Muller et al., 2008). Cette "nouvelle maintenance " utilise des techniques de prévision des pannes (par exemples : l'analyse des vibrations ou des huiles). Ce stade ultime de la maintenance, dite "prédictive " ou "préventive conditionnelle ", permet de remplacer des composants juste avant leur rupture (Muller, 2005). Le passage de la maintenance curative à la maintenance prédictive n'est cependant pas systématique. Il vise moins à minimiser les coûts de maintenance qu'à les optimiser en fonction des objectifs de production. Il peut ainsi être économiquement rentable d'appliquer une maintenance préventive systématique à un processus de production particulier (pour un produit phare à forte marge bénéficiaire par exemple). La maintenance curative peut s'avérer la seule «économiquement acceptable » pour un autre produit ou équipement sur lequel l'entreprise dispose de l'expérience et l'habitude de réparer des pannes. Les entreprises opèrent donc des choix parmi les différents types de maintenance, en fonction de nombreux critères techniques, économiques, internes et externes tels que la fréquence des défaillances cycliques ou aléatoires des équipements, les aptitudes et compétences du personnel de maintenance ou des sous-traitants, les politiques et modes d'organisation du travail...

Comme dans les travaux de Valdez-Florez et al., (1989), Simeu-Abazi et al., (1999), Wang (2002), Castanier et al., (2003), Dieulle et al., (2003), Welte (2008), et Soro et al., (2010), nous montrons qu'il est possible de proposer à l'expert, responsable de la maintenance, un niveau estimé de dégradation du processus maintenu à partir d'une collecte d'informations «terrain ». Une suite d'évènements particuliers peut, éventuellement, informer l'expert d'une panne prochaine. Notre étude tente d'appréhender « cette signature ». Les travaux présentés dans cet article, contribuent à enrichir la panoplie des méthodes et outils pour la modélisation de la surveillance et de la maintenance conditionnelle des systèmes. Ils répondent ainsi à des préoccupations très actuelles dans le domaine de l'aide à la décision de 
maintenance. Nous proposons donc à l'expert deux méthodes d'estimation du niveau de dégradation du système maintenu. La première méthode consiste à utiliser une loi non paramétrique ainsi qu'une loi semi-paramétrique en vue d'estimer le niveau de dégradation du système et fournir une référence de comparaison par rapport à l'approche markovienne. La deuxième méthode consiste à utiliser une approche markovienne permettant d'apporter à l'expert des informations sur le niveau de dégradation du système. Toutes les propositions sont illustrées sur un cas concret correspondant à une situation industrielle réelle (système continu pour l'agroalimentaire).

\section{Contexte général en maintenance}

La fonction maintenance, dont la vocation est d'assurer le bon fonctionnement des outils de production, est maintenant une des fonctions stratégiques majeures dans les entreprises. La norme européenne NF EN 13306X 60-319 (AFNOR, 2001) définit en totalité cette fonction. De manière plus pragmatique, un système de production n'est performant que si sa finalité, les objectifs qui lui sont attribués, les résultats qu'il fournit et les moyens (financiers, stratégiques, technologiques et humains) qu'il met en œuvre, sont en parfaite cohérence (Chandler, 1989). D'autres définitions de ce concept peuvent être trouvées dans Tarondeau et al., (1998) et Thiétart et al., (2001). Par exemple, les résultats des performances des entreprises en agroalimentaire (fabrication des pains de mie, brioches...) ou en verrerie (fabrication des plats pour cuisiner, de verres pour boire...) dépendent essentiellement du rendement des machines qui fabriquent les produits.

\section{Mise en œuvre d'une politique de maintenance}

Dans la définition de la maintenance (Wikipedia), nous trouvons deux mots-clés : maintenir et rétablir. Le premier fait référence à une action préventive, le deuxième fait référence à l'aspect correctif. On peut résumer les différentes politiques de maintenance selon la Figure 1. Une synthèse des différentes stratégies de maintenance peut être trouvée dans la thèse de Castanier (2002) et l'article de Despujols (2004). Le choix d'une politique est parfois imposé, comme c'est le cas dans le nucléaire en France. Ainsi, l'optimisation de la maintenance par la fiabilité détermine la maintenance préventive optimale. Dans le cas où il n'y a pas de maintenance préventive, cela revient à attendre la défaillance, c'est-à-dire une maintenance corrective. De nombreuses études ont montré que les politiques de maintenance préventive (ou plutôt mixte) sont souvent les moins onéreuses sur le long terme. Tout arrêt de production étant coûteux, Thomas et al., (2006) proposent une sélection optimale de ces arrêts parmi l'ensemble des arrêts de production déjà planifiés dans le temps selon l'état de dégradation du système. La phase 
d'optimisation intègre des critères tels que la fiabilité, la maintenabilité et la durée des arrêts de production.

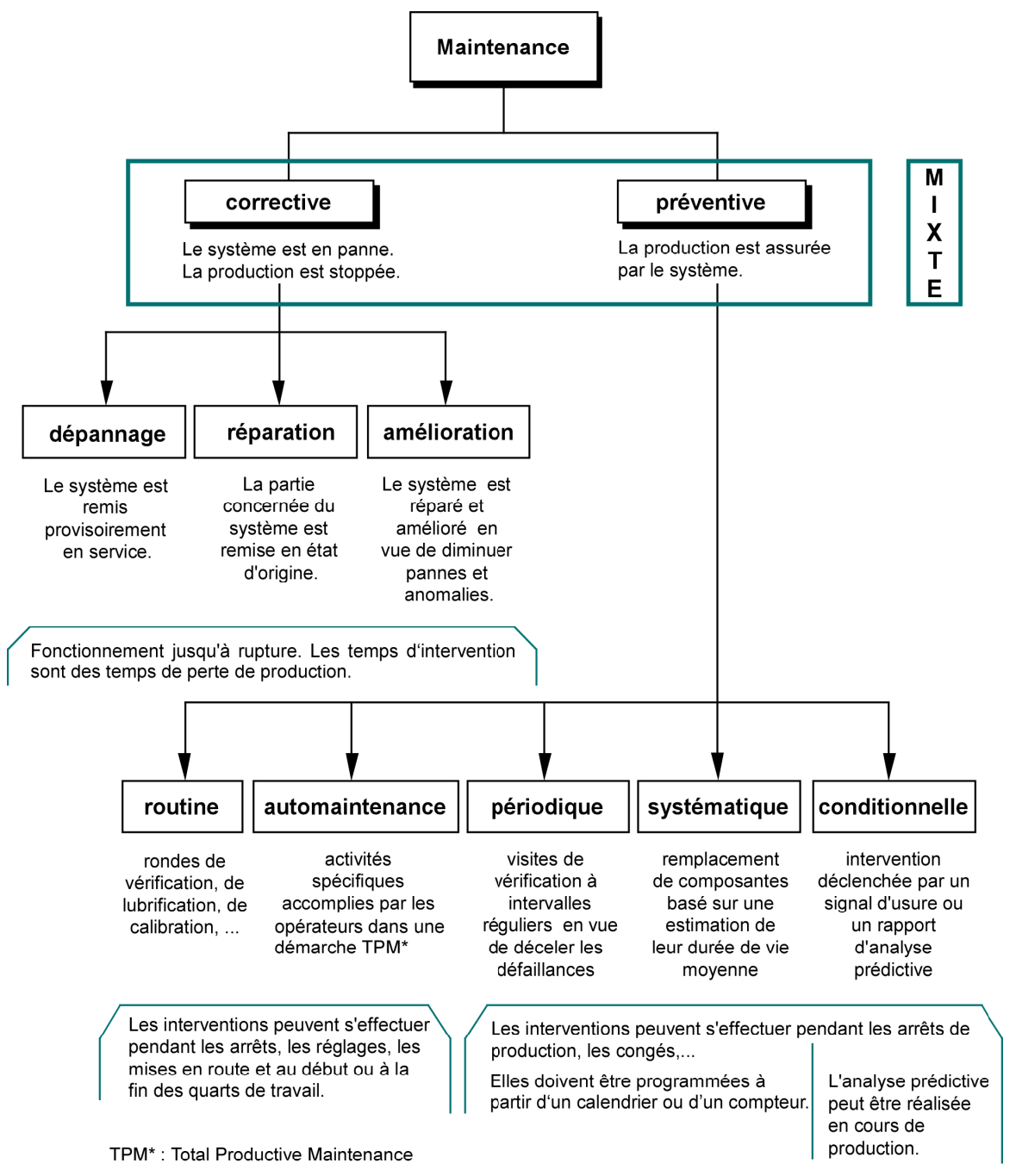

Figure 1. Synthèse de politiques de maintenance

Malgré qu'un équipement soit maintenu, une défaillance est possible. Cette défaillance peut être caractérisée par un taux $\lambda$ appelé taux de défaillance. Il est défini comme étant la probabilité conditionnelle que l'équipement tombe en panne entre l'instant $t$ et $t+\Delta t$ sachant qu'il a survécu jusqu'à l'instant $t$. Il peut aussi être défini comme la proportion de composants ayant survécu jusqu'à l'instant $t$ 
(équation [1]). Il représente également la vitesse d'arrivée de la panne (Monchy, 1991). On peut noter, que la variable «temps » doit être considérée comme une unité d'usage. En effet, dans le cas de certains dispositifs, il convient de considérer : une distance parcourue, un nombre de tours, un nombre de sollicitations...

$$
\lambda(t)=\lim _{\Delta t \rightarrow 0}\left(\frac{1}{\Delta t} \frac{R(t)-R(t+\Delta t)}{R(t)}\right)
$$

Avec :

- $\quad R(t)$ : fiabilité ou probabilité de survie d'un équipement déterminée à l'instant $t$,

- $\quad R(t+\Delta t):$ fiabilité ou probabilité de survie d'un équipement ayant survécu jusqu'à l'instant $t+\Delta t$.

L'évolution de la fonction d'intensité $\lambda(t)$ est déterminée par l'efficacité des opérations de maintenance. En pratique, une activité de maintenance est dite :

- minimale si l'état du système après réparation est le même que celui avant réparation : $\mathrm{ABAO}^{1}(\lambda(t)=\lambda ; \forall t \geq 0)$. L'intensité de défaillance est alors toujours égale à l'intensité initiale et ne dépend donc pas du passé du processus (Figure 2),

- $\quad$ parfaite si la maintenance remet le système à neuf : $\operatorname{AGAN}^{2}\left(\lambda_{t}=\lambda\left(t-T_{N t}\right)\right.$ avec $T_{N t-}$ la limite à gauche du processus stochastique $N(t)$ ), (Figure 2). Le processus stochastique $N(t)$ qui correspond à la maintenance minimale est un processus de Poisson. Par conséquent, si nous connaissons la loi de fiabilité du système, nous connaissons les paramètres du processus. Ce type de problème, envisagé à l'origine par Hastings (1969), a fait l'objet de nombreux développements ultérieurs incluant diverses variantes Phelps (1981), Muth (1997), Meier-Hirmer et al., (2008), réparation,

nuisible si l'état du système après réparation est moins fiable qu'avant

- efficace si le système est remis dans un état moins sujet aux pannes qu'avant réparation mais moins bien que le neuf,

- améliorative si l'état du système après réparation dispose d'une réduction du taux de panne par rapport au neuf.

\footnotetext{
${ }^{1}$ As Bad As Old

${ }^{2}$ As Good As New
} 

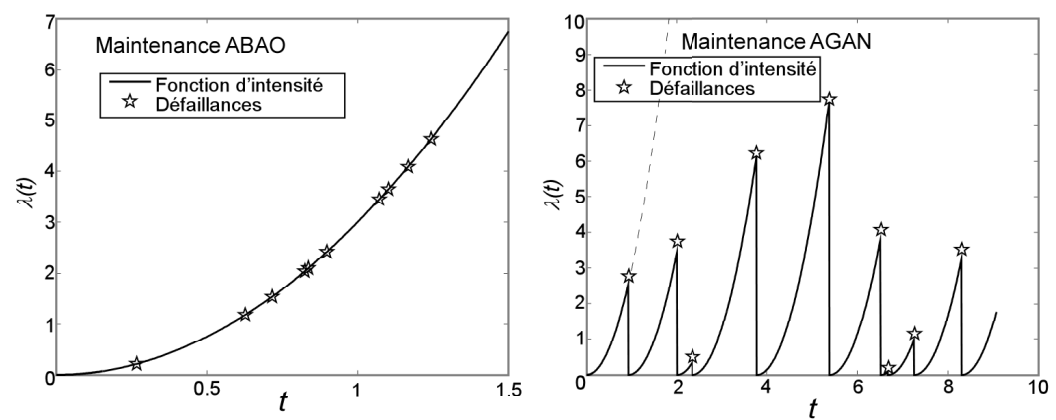

Figure 2. Fonction d'intensité pour une maintenance ABAO et AGAN (Basile, 2007)

\section{Etude de la fiabilité du processus maintenu}

Cette étude de la fiabilité est très souvent utilisée par l'expert. Nous présentons, dans cette section, quelques distributions de vie qui interviennent le plus fréquemment dans l'analyse des données de vie et qui sont communes à plusieurs champs disciplinaires. Nous parlerons en particulier des lois continues. Nous énoncerons les principales propriétés de ces lois (densité de probabilité, fonctions de fiabilité et taux de défaillance), Villemeur, (1988), Birolini, (1994). Les modèles de survie historiquement utilisés sont des modèles paramétriques et des modèles semiparamétriques à risques proportionnels (Bertholon et al., 2006).

Une maintenance optimale est reliée à une prévision de la durée de vie des composants de ces systèmes, prévision qui se fonde en premier lieu sur une analyse fine des données de retour d'expérience (ou REX). Plus précisément, l'étude de la durée de vie de tout système se scinde en deux alternatives distinctes, qu'il est important de ne pas confondre et qui nécessite un travail important de validation de ce REX :

- La fiabilité est l'aptitude d'un système à accomplir la fonction à laquelle il est dévolu, dans des conditions d'utilisation données et pendant un intervalle de temps donné (NF X 60-500, 1988), (NF X, 60-510, 1986) et (NF X, 60-520, 1988). L'ingénieur fiabiliste cherche à déduire de l'évolution temporelle de cette fiabilité une future stratégie de maintenance. Le type de REX utilisé est fonctionnel,

- La durabilité est l'aptitude d'un système à accomplir cette fonction, dans des conditions d'utilisation et de maintenance données, jusqu'à ce qu'un état limite soit atteint (NF EN 13306X 60-319, 2001). On peut grossièrement résumer la démarche de l'ingénieur durabiliste à l'estimation de la durée de vie restante, lorsque le système est en cours de fonctionnement. Une telle étude cherche à 
prendre en compte la modification des conditions d'exploitation, le renouvellement des matériels, ... pour en déduire le temps de bon fonctionnement du système.

Il existe différentes lois permettant de caractériser la fiabilité d'un système : loi exponentielle, loi normale (Laplace-Gauss), loi Log-normale (ou de Galton), loi de Weibull, de Bertholon. Pour exemple, la distribution dite de Weibull permet de représenter les trois périodes de la vie d'un dispositif décrites par la courbe en baignoire (jeunesse ou rodage, vie utile ou phase de maturité, vieillissement ou fin de vie (Villemeur, 1998)).

\subsection{Etude non paramétrique : estimateur de Kaplan-Meier}

La méthode de Kaplan-Meier, utilisée dans un champ disciplinaire large (Pacaut et al., 2007), (Cheuk-Kit et al., 2007), permet d'estimer les fonctions de survie, sans que les intervalles de temps ne soient nécessairement réguliers, contrairement à la méthode des tables actuarielles ${ }^{3}$. Les courbes de survie permettent d'analyser l'évolution de l'effectif d'une population donnée dans le temps. Cette technique (appelée aussi estimateur produit limite) est utilisée pour l'analyse de données de survie, qu'il s'agisse d'individus (recherche sur le cancer par exemple), ou de produits (résistance au temps d'un outil de production). Le premier type d'information est appelé "données évènement », tandis que le second est appelé «données censurées ». Soit $S(t)$, la fonction de survie et $\left(y_{1}, \ldots y_{n}\right)$ les données, l'estimateur empirique sans biais de Kaplan-Meier se formule de la manière suivante :

$$
S_{n}(t)=\prod_{i \in\{1, \ldots, n\}, y_{i}<t}\left(\frac{n-i}{n-i+1}\right)^{\delta_{i}}
$$

où $\delta_{i}=1$ si $y_{i}$ est une donnée non censurée ( 0 sinon).

\subsection{Etude semi-paramétrique : analyse d'un modèle à risques proportionnels de Cox}

Un modèle semi-paramétrique cherche à estimer la fonction de survie en tenant compte de l'influence des facteurs exogènes et sans faire aucune hypothèse a priori sur la forme de la distribution de base. Le modèle de régression de Cox est une méthode utile lorsqu'on veut déterminer l'impact de variables explicatives sur le temps de survie d'un processus économique (Gouriéroux, 1989), d'un patient dans le cadre d'une étude médicale (Breslow, 1973), d'un système (Kalbfleisch et al., 2002)...

Ce modèle développé par Cox (1972) est basé sur une estimation par maximum de vraisemblance dite partielle. Le principe du modèle de Cox qui se rapproche des

\footnotetext{
${ }^{3}$ Cette méthode regroupe les observations par intervalles aléatoires ou définis au préalable. Elle permet d'estimer et d'obtenir une représentation du taux de hasard.
} 
méthodes de régression classique est de relier la date d'arrivée d'un évènement à des variables explicatives. Par exemple, dans le domaine médical, on cherche à évaluer l'impact d'un pré-traitement sur le temps de guérison d'un patient. Cette approche se distingue par son pouvoir d'estimer la relation entre le taux de défaillance et les variables explicatives sans mettre aucune hypothèse sur la forme de la fonction de survie de base. Elle est basée sur l'hypothèse des risques proportionnels (le risque instantané de survenue de l'évènement considéré s'écrit comme le produit d'une fonction qui dépend du temps et d'une fonction qui dépend uniquement des caractéristiques du sujet étudié) et s'applique à toute situation où l'on étudie le délai d'occurrence d'un évènement. Le modèle de Cox est un modèle à risque proportionnel, dont le risque de base n'a pas de forme spécifiée et dont le risque relatif a une forme exponentielle.

Dans les modèles à risque proportionnel, le risque instantané s'écrit :

$$
\lambda(t \mid z, \theta)=\lim _{d t \rightarrow 0} \frac{1}{d t} P(t<T \leq t+d t \mid T>t, Z=z)=\alpha_{0}(t) f_{\beta}(z)
$$

avec :

- $\quad Z=\left(Z_{1}, \ldots Z_{p}\right)^{T}:$ un vecteur de covariables,

- $\quad \theta$ : les paramètres estimés en deux composants : un risque de base $\alpha_{0}$ (le risque de base, inconnu, indépendant de $Z$ ), et une fonction $\phi(z, \theta)$,

- $\quad \beta$ : le paramètre de régression, inconnu,

- $\quad f_{\beta}(z)$ : le risque relatif, indépendant du temps.

Par exemple : le ratio des risques pour deux individus est indépendant du temps. Les variables explicatives $Z_{1}, \ldots Z_{p}$, quantitatives ou qualitatives sont appelées facteurs pronostics (âge, sexe, traitement, ...). Cox (1972) a proposé comme modèle de survie pour le sujet $i$ :

$$
\lambda\left(t \mid Z_{i}\right)=\alpha_{0}(t) \exp \left(\beta_{1} Z_{i, 1}+\ldots, \beta_{p} Z_{i, p}\right)=\alpha_{0}(t) \exp \left(\beta^{T} Z_{i}\right)
$$

où $\alpha_{0}(t)$ est une fonction quelconque qui ne dépend que du temps (risque de base) et $\beta_{1}, \beta_{2}, \ldots \beta_{p}$ sont des constantes avec :

- $\quad \alpha_{0}(t)$ (le risque de base inconnu, indépendant de $Z_{i}$ ),

- $\quad \beta=\left(\beta_{1}, \ldots \beta_{p}\right)^{T}$ est le paramètre de régression inconnu,

- $\quad \exp \left(\beta^{T} Z_{i}\right)$ (le risque relatif, indépendant du temps),

- $\quad Z_{i}=\left(Z_{i, 1}, \ldots Z_{i, p}\right)$ est le vecteur des variables indépendantes. 
Si l'on note $\lambda_{0}$ la fonction de survie de base associée à $\alpha_{0}$, nous avons la relation suivante: $\lambda\left(t \mid Z_{i}\right)=\left[\lambda_{0}(t)\right] \exp \left(\beta^{T} Z_{i}\right)$, ce qui permet d'obtenir une estimation de $\lambda$ connaissant l'estimation du vecteur $\beta$. Pour estimer les composantes du vecteur $\beta$ à partir d'un échantillon ordonné $\left(y_{(1)}, \ldots y_{(n)}\right)$, on calcule la fonction de vraisemblance partielle de Cox qui n'est autre que (s'il n'y a pas de données censurées) :

$$
L\left(y_{(1)}, \ldots y_{(n)} ; \beta\right)=\prod_{i=1}^{n} \frac{\exp \left(\beta^{T} Z_{i}\right)}{\sum_{k \in R\left(y_{(i)}\right)} \exp \left(\beta^{T} Z_{k}\right)}
$$

A noter, que cette fonction de vraisemblance partielle reste identique dans le cas de données censurées à droite.

A noter, par exemple que :

- $\quad \exp \left(\beta_{i}\right)$ : taux relatif des sujets pour lesquels $Z_{i}=1$ par rapport à ceux pour lesquels $Z_{i}=0$, toutes choses égales par ailleurs,

- $\exp \left(\beta_{i}\right)>1$ : effet néfaste ; $\exp \left(\beta_{i}\right)=1$ : pas d'effet $; \exp \left(\beta_{i}\right)<1$ : effet protecteur.

\section{Tentative d'établissement d'un système d'aide à la décision par une approche markovienne : utilisation de modèles de Markov cachés}

Les modèles stochastiques sont des représentations de systèmes dynamiques basées sur les probabilités. L'étude des processus stochastiques a commencé au 17ème siècle avec Pascal et s'est poursuivie au XXème siècle grâce à un mathématicien Russe, Markov Andreï Andreïevitch. Son étude statistique du langage l'a conduit à formuler l'hypothèse markovienne, qui peut se résumer ainsi : «L'évolution future d'un système ne dépend que de son état présent ». Autrement dit, cette hypothèse implique que l'état courant du système contient toute l'information apportée par le passé. C'est donc une hypothèse très forte. En pratique, cette condition est rarement satisfaite. Cependant, l'approximation par ces modèles markoviens offre malgré tout de bons résultats de modélisation.

L'analyse des risques des systèmes dynamiques par une approche classique markovienne ne considère que deux états (Marche/Panne (Arrêt)). Entre l'état de marche parfaite et l'état de panne totale, les systèmes industriels présentent généralement un grand nombre d'états dégradés qui permettent d'assurer un service, même si celui-ci n'est pas égal à $100 \%$. "Ces états dégradés sont donc à prendre en compte pour évaluer correctement le niveau de service des systèmes industriels 
et cela est plus particulièrement vrai en ce qui concerne les systèmes de production » (Signoret, 2005).

Supposons que les évènements qui précèdent une panne soient souvent récurrents. Une suite d'évènements particuliers peut, éventuellement, être annonciatrice d'une panne prochaine. Quelques exemples connus peuvent illustrer notre hypothèse. En mécanique, un bruit, une vibration précèdent souvent la rupture. Une baisse de performances traduit une ou des anomalies. En informatique, un déplacement inopiné du pointeur ou de la souris, un ralentissement, un dysfonctionnement d'une application comme un navigateur Internet trahissent souvent la présence d'un virus sur l'ordinateur.

Notre étude a pour objet d'appréhender « cette signature » à l'aide d'un modèle de Markov caché. Le processus caché correspondra à l'état du système (ou soussystème) et les observations seront les informations observables sur le système telles que les différentes interventions de maintenance (Figure 3).

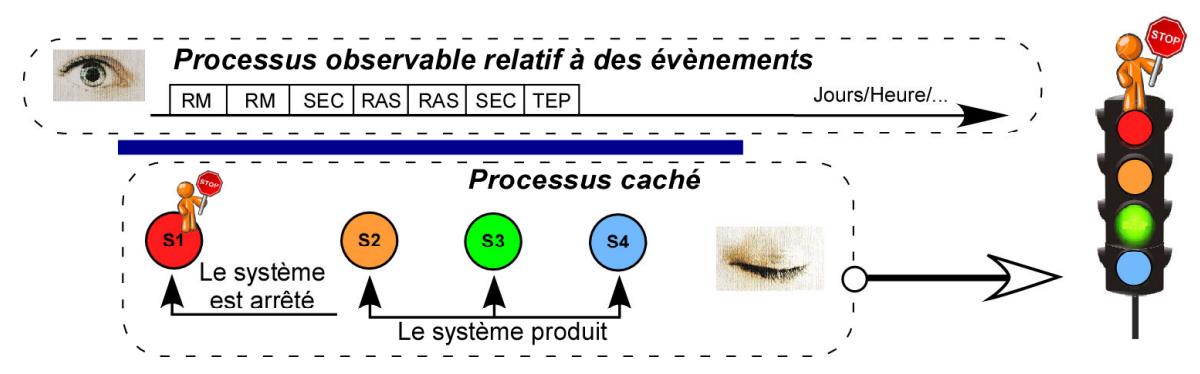

Figure 3. Processus observable et processus caché

Nos travaux tentent de montrer qu'il est possible de modéliser le niveau de dégradation d'un processus dit «continu» et que ceux-ci, complémentaire aux travaux de Zille et al., (2007), peuvent apporter une aide à la décision dans l'organisation et l'évaluation quotidienne de la maintenance (Figure 4).

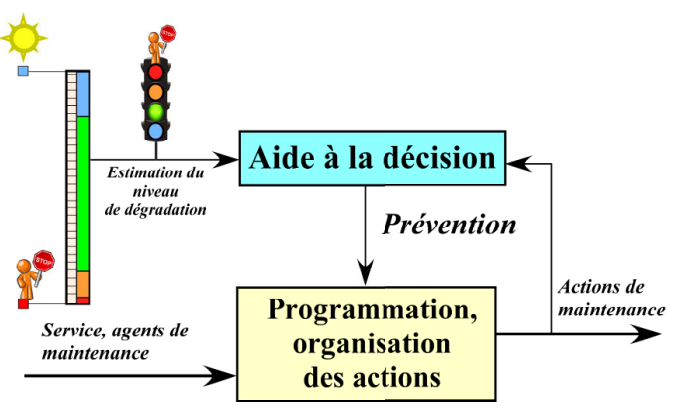

Figure 4. Objectif des travaux initiés 


\subsection{Approches markoviennes cachées}

Les modèles de Markov cachés (MMC) sont une famille d'outils mathématiques probabilistes parfaitement adaptés à la modélisation de séquences temporelles. C'est au début des années 70 que les modèles markoviens cachés ont véritablement connu leur essor en traitement du signal, essentiellement dans le cadre de la reconnaissance automatique de la parole. Ceci s'explique en partie par la découverte d'une procédure générale d'ajustement des paramètres des modèles aux caractéristiques du signal à reconnaître (Baum et al., 1966) et d'un algorithme de décodage (Viterbi, 1967) utilisé en théorie de l'information puis adapté par la suite au décodage des états cachés de la chaîne. Depuis lors, moyennant l'introduction de nouveaux éléments de modélisation, le cadre théorique des modèles markoviens cachés s'est considérablement élargi tandis que les approches par MMC se sont généralisées à d'autres domaines d'application : les travaux en reconnaissance de la parole (Schalapbach et al., 2004); traitement d'images (Aupetit, 2005); biosciences (Grundy et al., 1997) ; reconnaissance de l'écriture (Avila, 1996)... Les modèles de Markov cachés, étant des modèles de Markov stationnaires, modélisent des phénomènes aléatoires. On suppose que ceux-ci sont composés, à un premier niveau, d'un processus aléatoire de transition entre des états inobservables (les états cachés) et, à un second niveau, d'un autre processus aléatoire qui, dans chaque état, engendre des valeurs observables (appelées observations).

L'objectif de cet article n'est pas de présenter de façon détaillée les MMCs. Les lecteurs intéressés pourront se référer à Rabiner (1989) et Aupetit (2005) pour plus de détails. Un modèle de Markov caché discret correspond à la modélisation de deux processus stochastiques : un processus caché parfaitement modélisé par une chaîne de Markov discrète et un processus observé dépendant des états du processus caché. Soit $\mathbb{S}=\left\{s_{1}, \ldots s_{N}\right\}$ l'ensemble des $N$ états cachés du système. Soit $S=\left(S_{1}, \ldots S_{T}\right)$ un $T$-uple de valeurs aléatoires définies sur $\mathbb{S}$. Soit $\mathbb{V}=\left\{v_{1}, \ldots v_{L}\right\}$ l'ensemble des $L$ symboles émissibles par le système. Soit $V=\left(V_{1}, \ldots V_{T}\right)$ un $T$-uple de valeurs aléatoires définies sur $\mathbb{V}$. Un modèle de Markov caché discret du premier ordre est alors défini par les probabilités suivantes :

- les probabilités d'initialisation des états cachés: $P\left(S_{1}=S_{i}\right)$,

- les probabilités de transition entre états cachés: $P\left(S_{t}=s_{j} \mid S_{t-1}=S_{i}\right)$,

- les probabilités d'émission des symboles dans chaque état caché: $P\left(V_{t}=v_{j} \mid S_{t}=s_{i}\right)$.

Un modèle de Markov caché stationnaire du premier ordre $\lambda$ est totalement défini par le triplet de matrices $(A, B, \Pi)$. Les relations de dépendance entre les différentes variables aléatoires d'un MMC sont représentées sur la Figure 5 (Aupetit, 2005). 


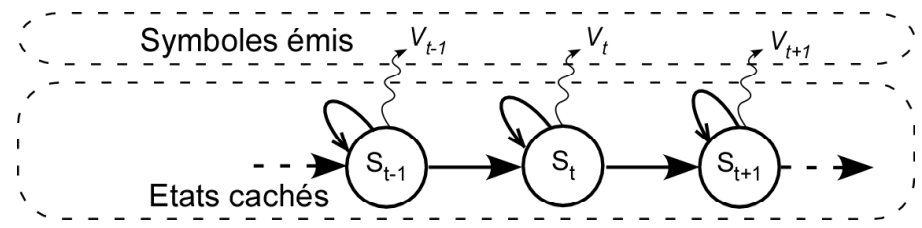

Figure 5. Etats cachés et symboles émis dans un $M M C$

On note $Q=\left(q_{1}, \ldots, q_{T}\right) \in \mathbb{S}^{T}$ une séquence d'états cachés et $O=\left(O_{1}, \ldots, O_{T}\right) \in \mathbb{V}^{T}$ une séquence de symboles observés. La probabilité de réalisation simultanée de la séquence d'états cachés $Q$ et de la séquence d'observations $O$ par rapport au MMC $\lambda$ est alors :

$$
P(V=O, S=Q \mid \lambda)
$$

La matrice de distribution des probabilités de transition entre états est caractérisée par :

$$
A=\left\{a_{i j}=P\left(S_{j} \mid S_{i}\right)\right\} ; \sum_{j=1}^{N} a_{i j}=1
$$

La matrice de distribution des probabilités de génération des symboles est caractérisée par :

$$
B=\left\{b_{i}\left(o_{T}\right)=P\left(o_{T} \mid S_{i}\right)\right\} ; \sum_{j=1}^{N} b_{i}\left(o_{j}\right)=1
$$

La matrice de distribution des probabilités initiales est caractérisée par :

$$
\Pi=\left\{\pi_{i}=P\left(S_{i}\right)\right\} ; \sum_{i=1}^{N} \pi_{i}=1
$$

\subsection{Outils utilisés}

La mise en œuvre d'un MMC nécessite l'usage de plusieurs outils pour les phases d'apprentissage et de décodage. Cette étude a nécessité de résoudre les deux problèmes classiques suivants :

- l'adaptation optimale du modèle $\lambda$ aux données initiales (l'apprentissage) : comment ré-estimer les paramètres du modèle $\lambda$ pour maximiser $P(V=O \mid \lambda)$ sur la base d'un ensemble de séquences d'observations? Deux méthodes différentes d'apprentissage ont été considérées.

- à partir d'une séquence d'observations, comment estimer la séquence d'états la plus probablement suivie? (décodage). Deux méthodes différentes de décodage ont été considérées. 


\subsubsection{Apprentissage du modèle : algorithme de Baum-Welch}

On parle d'apprentissage supervisé du MMC lorsque son architecture (sa topologie) est connue (Figure 7). L'algorithme d'apprentissage le plus communément utilisé est l'algorithme de Baum-Welch (Baum, 1972). Cet algorithme est dérivé de l'algorithme EM (Expectation Maximization). Il apporte une solution au problème d'apprentissage avec le critère du maximum de vraisemblance. Pour une séquence de $T$ observations, ce critère consiste à rechercher le MMC $\lambda^{*}$ qui a la plus grande probabilité d'engendrer la séquence $O$. On cherche donc à maximiser $P(V=O \mid \lambda)$. Cet algorithme est une procédure d'estimation itérative des matrices $A, B$ et $\Pi$ à partir d'un MMC initial. Il converge vers un optimum local de la fonction de vraisemblance. En appliquant cet apprentissage avec différents modèles initiaux, il est possible de se rapprocher de l'optimum global, c'est-à-dire d'obtenir un modèle presque optimal pour le critère considéré.

On obtient après exécution de l'algorithme, un MMC $\lambda^{*}$ tel que :

$$
P(O=O \mid \lambda) \approx P\left(O=O \mid \lambda^{*}\right)
$$

\subsubsection{Apprentissage du modèle : algorithme segmental k-means}

Parmi l'ensemble des critères utilisés pour l'apprentissage d'un MMC, le critère de «segmental K-means" se détache des autres. Pour ce critère, on cherche à maximiser la probabilité $P\left(V=O, S=Q^{*} \mid \lambda\right)$, avec $Q^{*}=\left(q_{1}^{*}, \ldots q_{T}^{*}\right) \in \mathbb{S}^{T}$ vérifiant, pour tout $t=1 \ldots T$, l'équation : $Q^{*}=\underset{Q \in \mathbb{S}^{T}}{\operatorname{argmax}} P(V=O, S=Q \mid \lambda)$. Cette probabilité correspond à la séquence d'états cachés qui a le plus probablement engendré la séquence telle que calculée par l'algorithme de Viterbi (1967). Tout comme l'algorithme de Baum-Welch, cet algorithme ne permet de converger que vers un optimum local. Une démarche utilisant différents modèles initiaux permet d'obtenir un modèle presque optimal. Aupetit (2005) propose une présentation détaillée de cet algorithme.

\subsubsection{Lissage des modèles}

L'une des propriétés des deux modes d'apprentissage précédents est de préserver la structure initiale des modèles. Lorsqu'une probabilité du modèle initial est nulle, alors la probabilité correspondante du modèle obtenu par l'apprentissage reste nulle. Il est cependant important de noter qu'une probabilité non nulle dans le modèle initial peut devenir nulle dans le modèle obtenu par l'apprentissage. Ce phénomène peut se produire lorsque certains symboles observables n'apparaissent pas dans la base de données d'apprentissage. La présence dans une nouvelle séquence, d'une seule occurrence de symbole absent lors de l'apprentissage, provoque une non reconnaissance par le MMC : la vraisemblance est nulle. Pour gérer ce problème, on introduit une étape de lissage des probabilités après l'apprentissage. Pour chacune 
des probabilités, non forcées à zéro par la structure du modèle, un epsilon est ajouté. Les contraintes de stochasticité des matrices sont obtenues en normant les sommes à 1. Ce lissage introduit une déformation du modèle optimal obtenu par l'apprentissage. La déformation introduite est négligeable tout en permettant de rendre le modèle «moins rigide». Nous distinguerons donc, dans la suite, les apprentissages lissés et les apprentissages non lissés.

\subsubsection{Décodage}

Une fois le modèle caractérisé, soit par l'algorithme de Baum-Welch, soit par l'algorithme segmental K-means, avec ou sans lissage, nous cherchons à estimer, pour de nouvelles séquences d'observations, les états probables du système. Ce qui peut également s'exprimer par «la recherche du chemin le plus probable (estimation de la partie cachée du modèle) : étant donnés la suite d'observations $O$ et un modèle $\lambda$, comment trouver une suite d'états $S=\left\{s_{1}, s_{2}, \ldots s_{N}\right\}$ qui soit optimale selon un critère correspondant aux attentes souhaitées?».

\subsubsection{Algorithme Variables Forward}

Pour un apprentissage avec l'algorithme de Baum-Welch, nous estimons l'état le plus probable à un instant donné en utilisant les variables Forward (Rabiner, 1989). Soit $\alpha_{t}(j)$ la probabilité de générer la séquence d'observations $O=\left\{O_{1}, O_{2}, \ldots o_{t}\right\}$ et de se trouver dans l'état $q_{t}$ à l'instant $t$, c'est-à-dire :

$$
\alpha_{t}(j)=P\left(o_{1}, o_{2} \ldots o_{t}, Q_{t}=s_{j} \mid \lambda\right)
$$

L'état le plus probable à l'instant $t$ est défini par : $\underset{j=1 . . k}{\operatorname{argmax}} \alpha_{t}(j)$.

\subsubsection{Algorithme de Viterbi}

Pour un apprentissage avec l'algorithme segmental $k$-means, nous estimons l'état le plus probable à un instant donné, en utilisant le dernier état du chemin optimal au sens de l'algorithme de Viterbi (Viterbi, 1967). La recherche de la séquence d'états cachés $Q^{*}$ qui a le plus probablement engendré une séquence d'observations $O$ consiste à résoudre :

$$
Q^{*}=\underset{Q \in \mathbb{S}^{T}}{\operatorname{argmax}} P(V=O, S=Q \mid \lambda)
$$

En conservant les notations précédentes, l'état le plus probable est défini par :

$$
\delta_{t}(j)=\max _{\left(q_{1}, \ldots, q_{t-1}\right) \in \mathbb{S}^{t-1}}\left\{P\left(S_{1}=q_{1}, \ldots, S_{t-1}=q_{t-1}, S_{t}=s_{j}, V_{1}=o_{1}, \ldots, V_{t}=o_{t} \mid \lambda\right)\right\}
$$

La Figure 6 décrit l'ensemble des procédures que nous avons adoptées pour effectuer l'ensemble des expérimentations sur le cas industriel. Dans tous les cas, 
l'expert pourra disposer d'éléments sur l'estimation du niveau de dégradation du système.

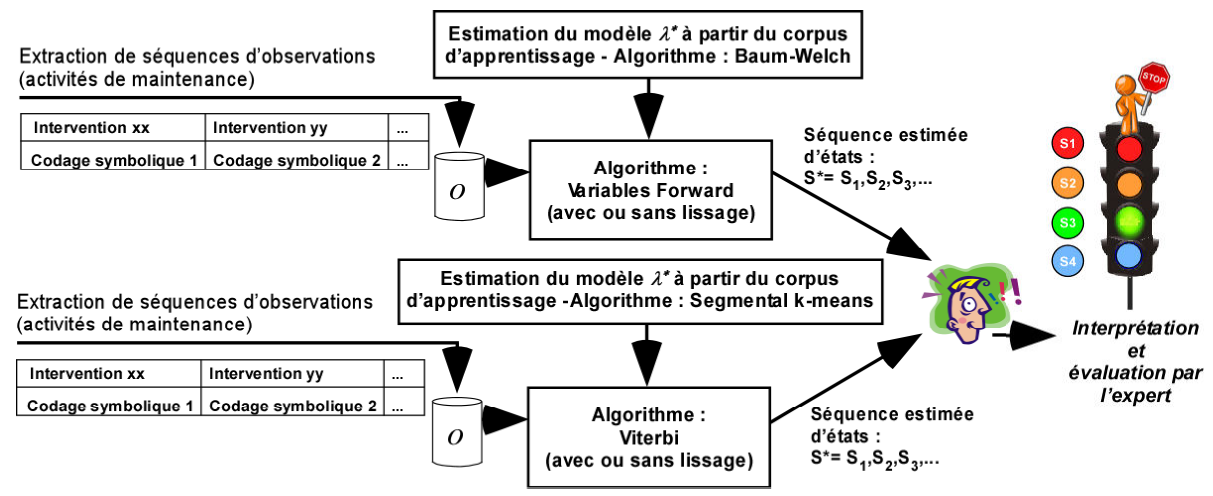

Figure 6. Modalités algorithmiques adoptées pour les différentes simulations

\subsection{Graphe d'états : choix de la topologie}

La topologie d'un MMC dépend directement des éléments non nuls de la matrice de transition notée $A$. En particulier, un MMC dont la matrice $A$ est pleine (aucun terme nul) est dite à connectivité totale : toutes les possibilités d'évolution sont permises. En pratique, il s'avère que la matrice $A$ est très rarement pleine car elle fixe la topologie et l'orientation future de l'analyse du modèle choisi. Avant de décrire les résultats sur les simulations effectuées, posons-nous la question suivante : Combien de niveaux de vigilance révélateurs et interprétables peut-on choisir ? Avec seulement trois niveaux de vigilance (ou états de vigilance), quel sens peut-on donner à l'état intermédiaire ? A partir de quatre niveaux, cette problématique disparaît. En augmentant le nombre de niveaux, on réduit la sensibilité de l'indicateur. Nous avons décidé d'adopter les pratiques reconnues et efficaces sur quatre niveaux (exemples : renforcement de la politique de prévention des risques liés aux inondations (Ministère de l'Écologie de l'Energie du Développement durable et de la Mer, 2006); (Ministère de l'intérieur, 2003); (Welte, 2008)...). La topologie du modèle présenté (Figure 7) montre que pour passer de l'état S4 à S1, il faut obligatoirement passer par S3 et S2. Pour l'ensemble des simulations effectuées, l'état $\mathrm{S} 1$ émettra exclusivement l'observation « $D E P$ » (situation qui conduit par définition à l'arrêt du système). Pour les autres états (S2, S3 et S4), chaque état émettra les autres observations possibles sauf l'observation « $D E P$ ». Les symboles utilisés en fonction des différentes observations sont explicités par la suite. Au démarrage d'une séquence, le système se trouve à l'état S4. On pourra également avoir à l'esprit le sens que nous avons voulu donner aux différents états : 
$\mathrm{S} 4$ «tout va bien », S3 «ça peut aller», $\mathrm{S} 2$ «ça va mal, attention », $\mathrm{S} 1$ «trop tard $»$.

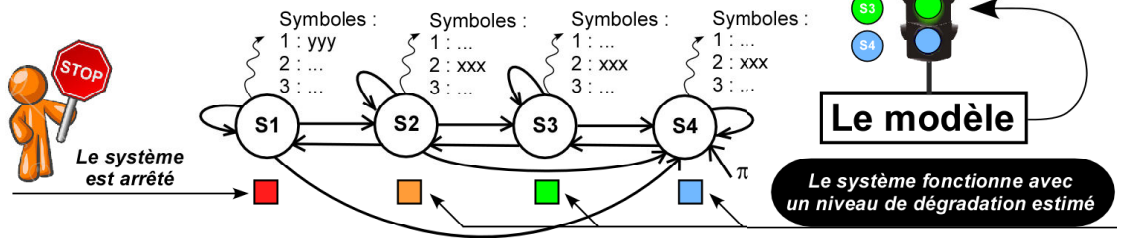

Figure 7. Modèle à topologie orientée à 4 états

\section{Etude d'un processus industriel dans le cadre d'une politique de maintenance préventive}

Nous présentons, dans ce paragraphe, le cas d'un processus continu de fabrication de pain de mie. Ce processus fonctionne toute l'année sans interruption en équipes organisées en $3 \times 8$. Pour chaque équipe, des bases de données sont disponibles et renseignées conformément aux activités de maintenance opérationnelle (Tableau 1) dans une politique de maintenance préventive. La Figure 8 décrit l'organisation du processus continu.

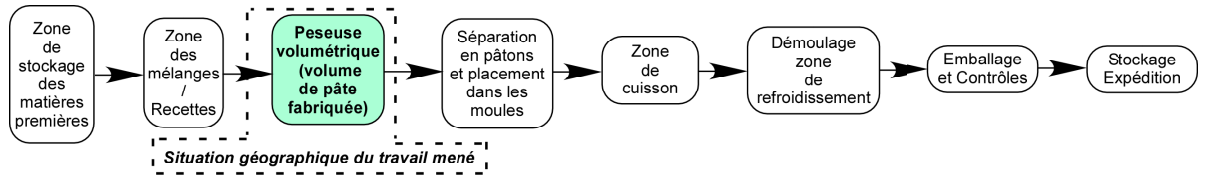

Figure 8. Principe d'organisation du processus continu étudié

L'arrêt d'un sous-système engendre l'arrêt total de production en amont de celui-ci. Une maintenance préventive est donc indispensable. Notre étude porte plus particulièrement sur le sous-système peseuse volumétrique. Le fonctionnement de cette peseuse volumétrique peut être assimilé au fonctionnement d'une seringue que l'on remplirait par un effet de dépression. L'ensemble des données traitées dans notre étude est issu des informations collectées sur les années 2005, 2006 et $1^{\text {er }}$ trimestre 2007. Le Tableau 2 décrit la signification des codes (activités 
opérationnelles de maintenance) au sein de l'entreprise. Dans la suite, nous étudions ces données dans le cadre de nos différentes approches.

\begin{tabular}{|c|c|c|c|c|c|c|c|c|c|c|c|}
\hline $\begin{array}{c}\text { Numéro dans la } \\
\text { liste }\end{array}$ & NOM & EQUIPE & DATE & LIGNE & $\begin{array}{c}\text { NOM DE } \\
\text { MACHINE }\end{array}$ & $\begin{array}{c}\text { TRAVAUX } \\
\text { EFFECTUES }\end{array}$ & $\begin{array}{c}\text { CODES } \\
\text { MAINTENANCE }\end{array}$ & TI (min) & $\begin{array}{c}\text { PIECES } \\
\text { CHANGEES }\end{array}$ & REF \\
\hline 1 & M.Dupond & AM & $03 / 01 / 2005$ & M2 & Peseuse & $\begin{array}{c}\text { la demande du } \\
\text { RL }\end{array}$ & & RM & 20 & & \\
\hline
\end{tabular}

Tableau 1. Exemple de consignation des activités opérationnelles en maintenance sur la peseuse M2

\begin{tabular}{|c|c|c|}
\hline Nature des & DEP (Dépannage/arrêt prod) & $\begin{array}{l}\text { SEC (SÉcurité) } \\
\text { Activite lié a al sécurtíé de la machine et consignations }\end{array}$ \\
\hline inte & RM (Réglage Machine) & RAN (Remise A Niveau / planifié) \\
\hline (é & $\begin{array}{l}\text { AU (Autre) } \\
\text { Cette activite peut être associée à de pefits travaux }\end{array}$ & NET (Nottoyage Machine) \\
\hline onserves) & OBS (Observation) & 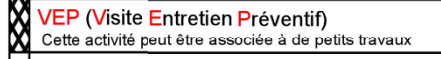 \\
\hline \begin{tabular}{|l|} 
Etat du processus \\
MARCHE
\end{tabular} & \begin{tabular}{|l|} 
TEP (Travaux Entretien \\
Préventif pas d'arrêt prod)
\end{tabular} & Ras pas dintervention \\
\hline
\end{tabular}

Tableau 2. Activités de maintenance recensées par le service maintenance

La base de notre travail s'est appuyée sur les données collectées en 2005 et 2006. Le Tableau 3 est un extrait des informations collectées, relatives aux activités de maintenance sur la période retenue (données complètes non censurées). A partir de ces données, nous avons effectué une étude statistique descriptive concernant le sous-système étudié.

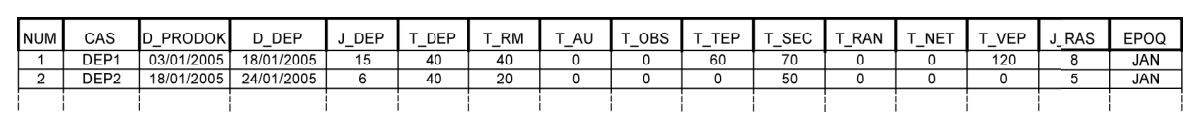

Tableau 3. Bilan des informations relatives aux activités de maintenance dans la base de données

Les définitions des entêtes du Tableau 3 sont : CAS : situations de dépannage, D_PRODOK : date production OK, D_DEP : date apparition d'une situation de dépannage, J_DEP : situations entre 2 dépannages (jour), T_xxx (temps passé dans l'activité en min) avec $\mathrm{xxx}$ : DEP, RM, AU, OBS, TEP, SEC, RAN, NET, VEP, J_RAS : temps passé en RAS (jour), EPOQ : époque de l'année (mois). 


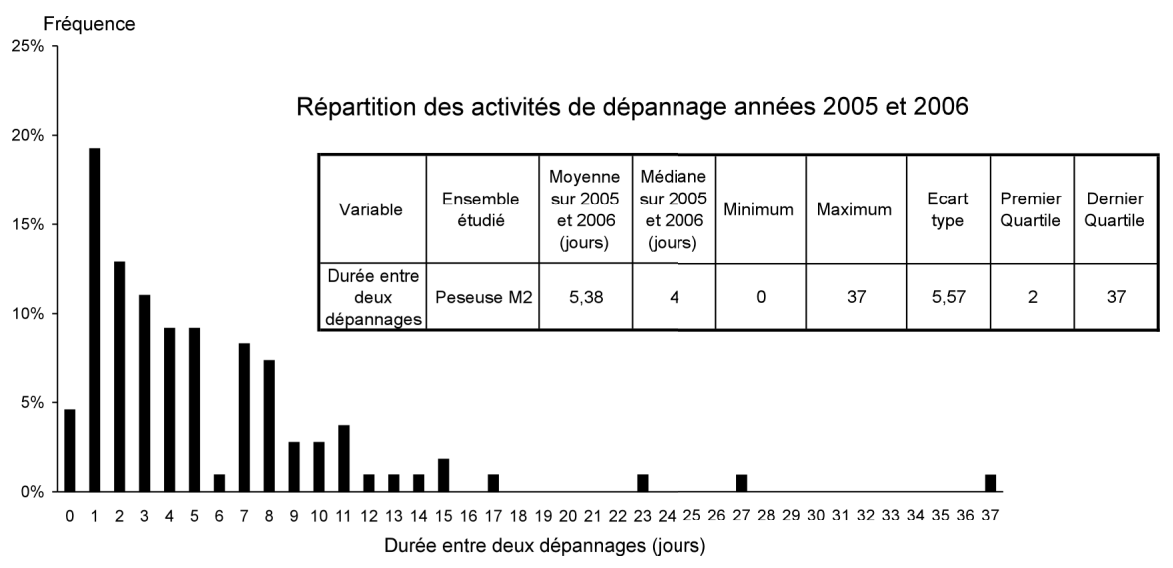

Figure 9. Répartition des pannes, mesures de tendance, de dispersion et d'emplacement

Lors de notre étude des durées de vie, nous tenons compte des situations où la peseuse est tombée en panne (nous avons choisi de n'adopter aucune censure dans le traitement des données). Après une analyse descriptive des durées de vie de la peseuse, nous aborderons une étude non paramétrique pour estimer sa fiabilité. Lors de l'étude des durées de vie de la peseuse, nous ne prenons en compte que les durées de fonctionnement (Figure 9). La durée moyenne entre deux situations de dépannage, où le système est arrêté, est de 5,38 jours. Elle inclut, entre deux situations de dépannage, toutes autres activités opérationnelles possibles en maintenance respectant la codification symbolique interne (RM, AU, OBS, ..., cf Tableau 2). L'histogramme (Figure 9) montre que le système a besoin d'être très fortement maintenu. Dans 19,27\% des cas, la panne arrive au bout d'une journée.

6.1. Résultats, par l'estimateur de Kaplan-Meier, de la fonction de survie du système

La Figure 10 donne la fonction de survie encadrée par un intervalle de confiance à $95 \%$. Par cette approche, l'expert dispose d'éléments qui lui indiquent que la dégradation du système suit une loi exponentielle (Figure 10). Pour le système continu étudié, ayant besoin d'être "fortement maintenu », on constate qu'après cinq jours de fonctionnement suite à un dépannage, celui-ci a 57\% de chance d'être déjà tombé en panne (estimation médiane du Tableau 5). 


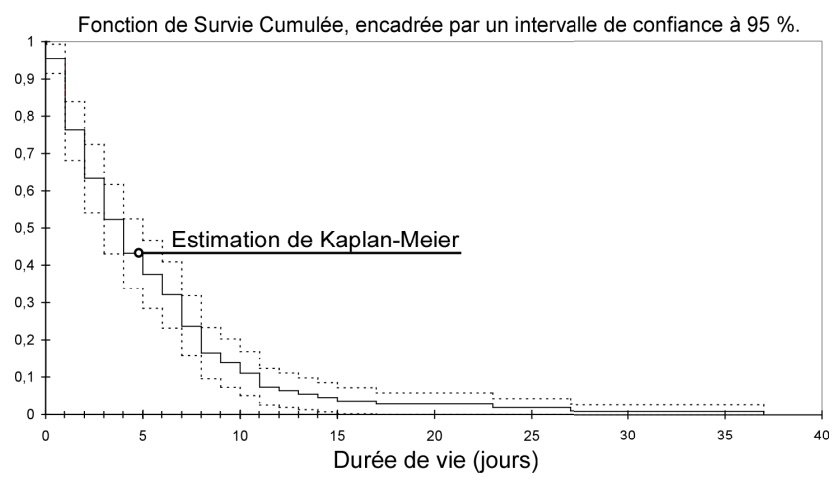

Figure 10. Fonction de survie par l'estimateur de Kaplan-Meier

\subsection{Résultats de l'analyse d'un modèle à risques proportionnels de Cox}

Les valeurs estimées (Tableau 4), dans la colonne «valeur», sont les estimations numériques des paramètres du modèle de Cox. Le terme $P_{r}>\chi^{2}$ est une $\mathrm{p}$-valeur dans le test de l'hypothèse $\mathrm{H}_{0}$ (paramètre $\left.=0\right)$, si cette $\mathrm{p}$-valeur est plus petite que 0,05 ( $\mathrm{si}$ on prend un risque de $5 \%$ ), alors le paramètre sera significativement différent de 0 . Ce terme $\left(P_{r}>\chi^{2}\right)$ est le seuil de rejet de l'hypothèse $\mathrm{H}_{0}$. Cette valeur est définie d'une part, par la statistique de Wald (calculée pour chaque variable) et d'autre part par, la valeur critique du $\chi^{2}$. Les valeurs $\exp \left(\beta_{i}\right)$ sont placées dans la colonne « Rapport de risque, Rapport de risque borne inf. et sup.».

Le Tableau 4 montre que seule la variable T_SEC a un impact sur le temps de survie du processus (temps entre deux situations de dépannage). Le coefficient $\exp \left(\beta_{i}\right)$, pour la borne sup. $(95 \%)$ est le seul $<1$ associé au terme $\left(P_{r}>\chi^{2}\right)<0,0001$. Nous pouvons conclure que plus le temps passé dans l'activité de maintenance rattachée à la sécurité (code SEC) sera « important», plus le temps de survie du processus sera potentiellement grand.

\begin{tabular}{|cccccccc|}
\hline Variable & Valeur & Ecart-type & Khi ${ }^{2}$ de Wald & $\mathrm{Pr}>\mathrm{Kh}^{2}$ & $\begin{array}{c}\text { Rapport de } \\
\text { risque }\end{array}$ & $\begin{array}{c}\text { Rapport de } \\
\text { risque Borne } \\
\text { inf. (95\%) }\end{array}$ & $\begin{array}{c}\text { Rapport de } \\
\text { risque Borne } \\
\text { sup. (95\%) }\end{array}$ \\
\hline T_DEP & 0,001 & 0,001 & 4,843 & 0,028 & 1,001 & 1,000 & 1,003 \\
T_RM & $-0,004$ & 0,005 & 0,586 & 0,444 & 0,996 & 0,987 & 1,006 \\
T_AU & 0,002 & 0,003 & 0,734 & 0,392 & 1,002 & 0,997 & 1,008 \\
T_OBS & $-0,007$ & 0,005 & 2,175 & 0,140 & 0,993 & 0,984 & 1,002 \\
T_TEP & 0,000 & 0,001 & 0,064 & 0,801 & 1,000 & 0,998 & 1,001 \\
\hline T_SEC & $-0,037$ & 0,009 & 18,976 & $<0,0001$ & 0,963 & 0,947 & 0,980 \\
\hline T_RAN & $-0,001$ & 0,001 & 1,138 & 0,286 & 0,999 & 0,996 & 1,001 \\
T_NET & $-0,008$ & 0,013 & 0,442 & 0,506 & 0,992 & 0,967 & 1,016 \\
T_VEP & $-0,002$ & 0,002 & 1,132 & 0,287 & 0,998 & 0,995 & 1,001 \\
\hline
\end{tabular}

Tableau 4. Coefficients de régression du modèle à risques proportionnels de Cox 
La Figure 11 décrit la fonction de survie. Nous pouvons constater que les résultats du test de Cox proposent des valeurs plus pessimistes pour la fonction de survie que dans le cas de l'estimateur de Kaplan-Meier (Figure 10).

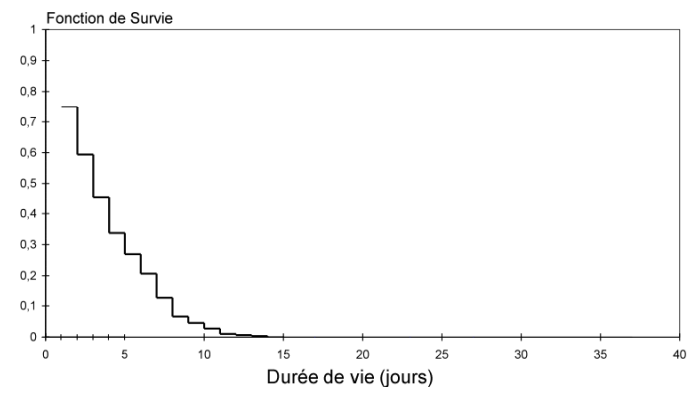

Figure 11. Fonction de survie par le modèle de Cox

\subsection{Premier bilan pour l'expert concernant l'étude des durées de vie}

Le Tableau 5 donne un bilan des différentes probabilités «d'être tomber en panne » à un instant donné. A partir de cette première approche, l'expert pourra ajuster son niveau de vigilance et décider ou non d'une intervention de maintenance en fonction de son retour d'expérience et de la probabilité d'être en panne par rapport à la dernière situation de dépannage.

\begin{tabular}{|c|c|c|c|c|c|c|c|c|c|c|c|c|}
\hline & \multicolumn{12}{|c|}{ Probabilités d'être tombé en panne à un instant donné sur le corpus des données 2005 et 2006} \\
\hline & à +1 jour & $\grave{a}+2$ jours & $\grave{a}+3$ jours & à +4 iours & $\grave{a}+5$ iours & à +6 iours & $\grave{a}+7$ iours & à +8 iours & à +9 jours & $\grave{a}+10$ jours & à+ 11 jours & $\grave{a}+12$ jours \\
\hline \multirow{2}{*}{$\begin{array}{c}\text { Estimation de } \\
\text { Kaplan-Meier avec } \\
\text { un intervalle de confiance } \\
\text { à } 95 \% \\
\end{array}$} & $1 \%$ & $15 \%$ & $27 \%$ & $38 \%$ & $48 \%$ & $53 \%$ & $59 \%$ & $68 \%$ & $76 \%$ & $79 \%$ & $83 \%$ & $88 \%$ \\
\hline & $8 \%$ & $32 \%$ & $45 \%$ & $57 \%$ & $66 \%$ & $72 \%$ & $76 \%$ & $84 \%$ & $91 \%$ & $93 \%$ & $95 \%$ & $97 \%$ \\
\hline $\begin{array}{l}\text { Estimation de } \\
\text { Kaplan-Meier }\end{array}$ & $4 \%$ & $23 \%$ & $36 \%$ & $47 \%$ & $57 \%$ & $62 \%$ & $67 \%$ & $76 \%$ & $83 \%$ & $86 \%$ & $89 \%$ & $92 \%$ \\
\hline $\operatorname{cox}$ & $25 \%$ & $41 \%$ & $54 \%$ & $66 \%$ & $73 \%$ & $79 \%$ & $87 \%$ & $93 \%$ & $95 \%$ & $97 \%$ & $98 \%$ & $99 \%$ \\
\hline
\end{tabular}

Tableau 5. Probabilités d'être tombé en panne à un instant donné

Nous avons montré que l'estimateur de Kaplan-Meier et le modèle de Cox (deux modèles statistiques non paramétrique et semi-paramétrique) peuvent apporter à l'expert un certain nombre d'éléments. En l'absence d'information a priori sur la forme de la fonction de survie, nous l'avons estimée d'abord par la méthode nonparamétrique de Kaplan-Meier. Puis, pour introduire des variables exogènes dans le modèle, nous avons étudié un modèle semi-paramétrique de Cox. Ces différentes expérimentations montrent que ce système a besoin d'être "fortement maintenu ». Cependant, les modèles de Kaplan-Meier et Cox ne proposent à l'expert que deux 
états pour définir l'état du système maintenu (Figure 12). Il n'y a pas d'état supplémentaire possible et le principal indicateur est le temps en dehors de corrélations possibles si elles existent. Nous avons montré qu'il existait dans le cas de la peseuse M2, une corrélation possible entre le temps passé dans l'activité «SEC » et le temps de survie du processus.
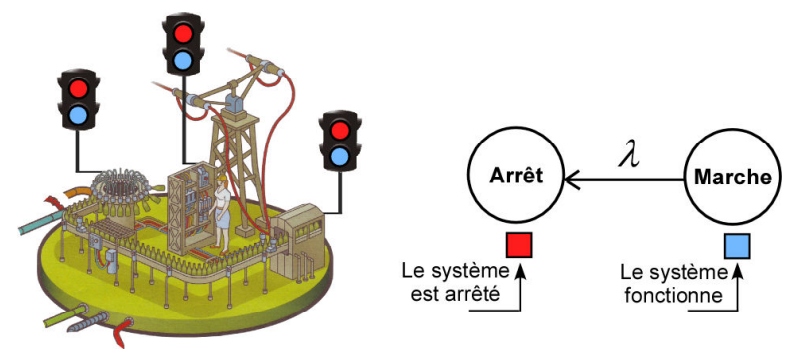

Figure 12. Modèles de Kaplan-Meier et Cox associés aux deux états d'un système

Plusieurs problèmes se posent :

- est-il possible de rajouter des états intermédiaires entre «marche» et «arrêt » (Figure 12)?

- Est-il alors possible d'anticiper l'arrivée d'une panne ?

- Comment choisir la «bonne» fréquence, la bonne cadence de collecte des informations, pour estimer de façon fiable et suffisamment tôt le niveau de dégradation du processus maintenu?

Nous présenterons dans la section suivante, le détail de notre approche utilisant les MMCs. Nous apporterons une réponse aux questions évoquées ci-dessus.

\subsection{Résultats obtenus par l'utilisation de modèles de Markov cachés}

6.4.1. Choix de la codification symbolique des observations et choix du critère d'insertions de symboles

La particularité du modèle de Markov caché est qu'il a besoin d'être «alimenté » en observations pour évoluer, afin de permettre une mise à jour du modèle à intervalle de temps plafonné. En l'absence d'évènement, une fois ce plafond atteint, un symbole particulier signifiant «Ras » (Rien à signaler (Figure 13)) est inséré dans la séquence initiale. Deux scénarios ont été étudiés : l'un avec une insertion avec un plafond à la journée ( 24 heures), l'autre avec un plafond à 6 heures (temps maximum entre deux activités de maintenance établi par rapport à l'ensemble de la base de données), sachant que la planification sur le terrain, par l'expert, des activités de maintenance, s'effectue à la journée. Cette approche d'insertions de symboles permettra donc d'actualiser l'état du modèle au moins une fois par jour. 


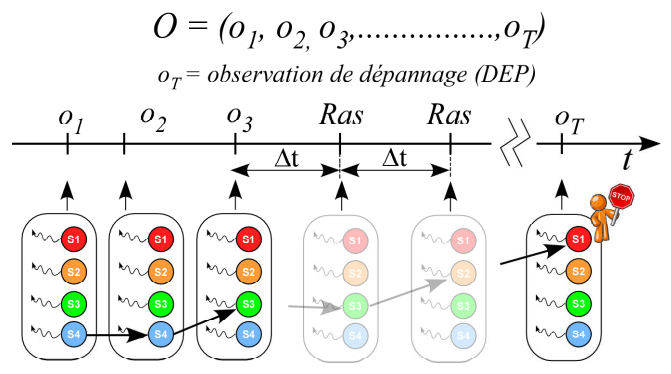

Figure 13. Séquence de symboles observés et séquence d'états cachés respectant le critère d'insertions de symboles

A partir du Tableau 2, nous avons codifié arbitrairement de 1 à 10 l'ensemble des différentes occurrences (activités de maintenance recensées par le service de maintenance associées et code «Ras» : pas d'intervention (Figure 14)).

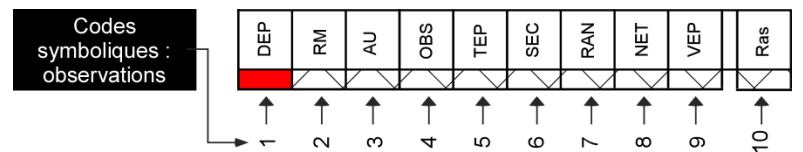

Figure 14. Présentation du choix de codification symbolique des différentes occurrences d'observations (peseuse M2)

6.4.2. Résultats en fonction du corpus d'apprentissage, du modèle choisi et $d u$ critère d'insertions de symboles

Notre approche consiste à donner "un sens très fort» à l'estimation de l'état S2 : ne pas détecter la panne, ni trop tard, ni trop tôt, ce qui pourrait engager sur le terrain des interventions inutiles. Les Figures 6 et 15 décrivent la procédure adoptée pour effectuer l'ensemble des expérimentations concernant la peseuse volumétrique.

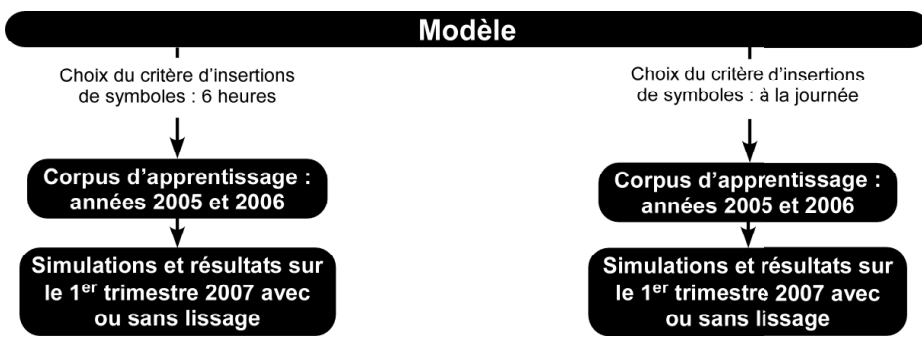

Figure 15. Procédures d'insertions de symboles adoptées pour effectuer les expérimentations 


\subsubsection{Bilan des différentes modélisations}

Avant de détailler l'ensemble des premiers résultats (Tableau 9), nous allons apporter un commentaire sur le contenu des différents entêtes d'un tableau type (Tableau 6). La première colonne (S1 / DEP) correspond à la capacité du modèle à estimer l'état $\mathrm{S} 1$, exclusivement par rapport à l'observation « $D E P$ ». La deuxième colonne (S3 ou S4 : 1 observation avant DEP) correspond à une non détection de l'état S2 (orange) avant S1. Dans ce cas, il n'y a aucune "alarme » (état S2) disponible pour l'expert. Malgré la topologie choisie, ce type de situations peut arriver, car à chaque nouveau symbole, c'est l'ensemble de la séquence qui est estimée. Le chemin le plus probable peut donc avoir changé. Les colonnes suivantes (Quantité successive de S2 avant S1) vont permettre de montrer que le modèle dispose d'une capacité à alerter l'expert plus ou moins rapidement (état S2) avant l'arrivée de la panne.

La dernière colonne (Quantité de S2 ne conduisant pas à S1) décrit la « sensibilité » du modèle à estimer l'état S2 trop en avance par rapport à l'arrivée de la panne (la Figure 16 montre sur un exemple que cette situation est possible).

\begin{tabular}{|l|c|c|c|c|c|c|}
\hline \multirow{2}{*}{$\begin{array}{c}\text { S1 } \\
\text { DEP ou S4 : } 1 \text { observation } \\
\text { avant DEP }\end{array}$} & \multicolumn{4}{|c|}{ Quantité successive de S2 avant S1 } \\
\cline { 2 - 7 } & 1 & 2 & 3 & $4-5$ & + de 5 \\
\hline
\end{tabular}

Tableau 6. Déclaration des entêtes

La situation optimale concernant les résultats serait (Tableau 7) : S3 ou S4 : 1 observation avant DEP $=0 \%$, quantité de $\mathrm{S} 2$ ne conduisant pas à $\mathrm{S} 1=0 \%$ avec un pourcentage élevé pour les premières cases avec l'entête : quantité successive de S2 avant $\mathrm{S} 1$.

\begin{tabular}{|c|c|c|c|c|c|c|c|}
\hline (s1 & \multirow{2}{*}{$\begin{array}{c}\text { S3 ou S4 : } 1 \text { observation } \\
\text { avant DEP }\end{array}$} & \multicolumn{5}{|c|}{ Quantité successive de S2 avant S1 } & \multirow{2}{*}{$\begin{array}{l}\text { Quantité de S2 ne } \\
\text { conduisant pas à S1 }\end{array}$} \\
\hline DEP & & 1 & 2 & 3 & $4-5$ & + de 5 & \\
\hline $100 \%$ & $0 \%$ & $100 \%$ & $0 \%$ & $0 \%$ & $0 \%$ & $0 \%$ & $0 \%$ \\
\hline
\end{tabular}

Tableau 7. Résultat idéal

La situation du Tableau 7, dans les différents cas testés, n'existe pas. Il nous faut donc trouver un compromis pour ne pas détecter la panne, ni trop tard (cas extrême où l'état S2 n'est pas détecté avant S1 (2) du Tableau 8)), ni trop tôt (occurrence d'états S2 successifs avant S1 (1) du Tableau 8)) ! Le cas (3) traduit la capacité du modèle à faire remonter l'estimation du niveau de dégradation du système de $\mathrm{S} 2$ vers S3 ou S4. Néanmoins, cette situation doit exister, mais doit être minimisée car elle traduit le niveau de sensibilité du modèle par rapport aux observations. Plus un 
modèle est sensible, plus il alertera de manière intempestive l'expert qui planifiera obligatoirement des interventions de maintenance inutiles.

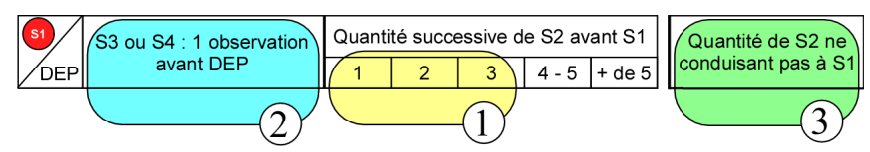

Tableau 8. Modalité de lecture du tableau

Le Tableau 9 présente un bilan complet des résultats concernant le modèle étudié pour les deux scénarios d'insertions. Ce modèle estime bien l'état «ARRET» en fonction de l'activité de maintenance rattachée (DEP) pour les deux scénarios d'insertions de symboles (Tableau 9 (a)) et Tableau 9 (b)). Nous constatons en général que le lissage apporte une meilleure qualité de résultats pour la condition (2).

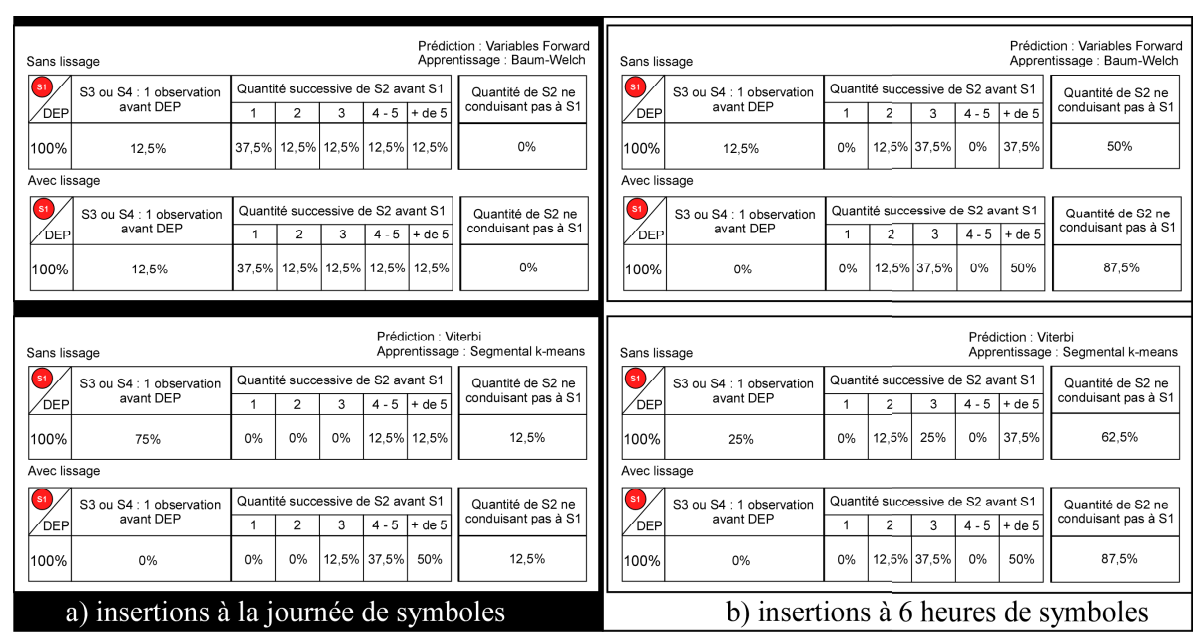

Tableau 9. Résultats des différentes simulations effectuées sur la période $1^{\text {er }}$ trimestre 2007

Compte tenu du compromis (capacité à détecter la panne) préalablement explicité nous pouvons faire ressortir du Tableau 9 (a) deux cas :

- cas $n^{\circ} 1$ : apprentissage du modèle avec l'algorithme de Baum-Welch suivi de prédictions avec lissage utilisant l'algorithme Variables Forward. Le modèle ne reconnaît pas un passage en S2 avant un passage en S1 (2) (12,5\% des cas). Sa capacité à détecter la panne (1) est bonne. $\mathrm{A}+3$ observations, on obtient $62,5 \%$ de chance de tomber en panne et $87,5 \%$ à +5 observations. D'autre part, nous pouvons constater que sa sensibilité est bonne (3) $=0 \%$ ). 
- $\quad$ cas $n^{\circ} 2$ : apprentissage du modèle avec l'algorithme de segmental k-means suivi de prédictions avec lissage utilisant l'algorithme de Viterbi. Le modèle reconnait un passage en S2 avant un passage en S1 (2) dans $100 \%$ des cas. Sa capacité à détecter la panne est nettement moins bonne que dans le cas précédent (1). A +3 observations, on obtient $12,5 \%$ de chance de tomber en panne. D'autre part, nous pouvons constater que sa sensibilité est non nulle (3) $=12,5 \%$ ). Dans ces conditions (3), l'expert aurait été alerté de manière inappropriée.

Le Tableau 9 (b) (scénario 2 : insertions de symboles toutes les 6 heures. Les modalités d'insertions respectent les explications présentées dans la Figure 13 avec $\Delta \mathrm{t}=6$ heures) appelle à plusieurs commentaires. Cette partie de tableau montre clairement que la prédiction évolue beaucoup entre les états S2, S3 et S4 avant d'arriver en S1 (3)). Dans 87,5\% des cas, S2 n'est pas un état qui précédera exclusivement l'état S1 (3)). Le modèle est trop sensible. D'autre part, il y a un étalement des états $\mathrm{S} 2$ dans le temps avec $50 \%$ des cas où $\mathrm{S} 2$ se cumule plus de 5 fois avant la détection de S1 (1). Nous pouvons également constater que le lissage apporte une meilleure qualité de résultats pour la condition S3 ou S4 : 1 observation avant DEP. La Figure 16, montre sur un exemple, les différences majeures qui existent entre les deux scénarios d'insertions de symboles utilisées.

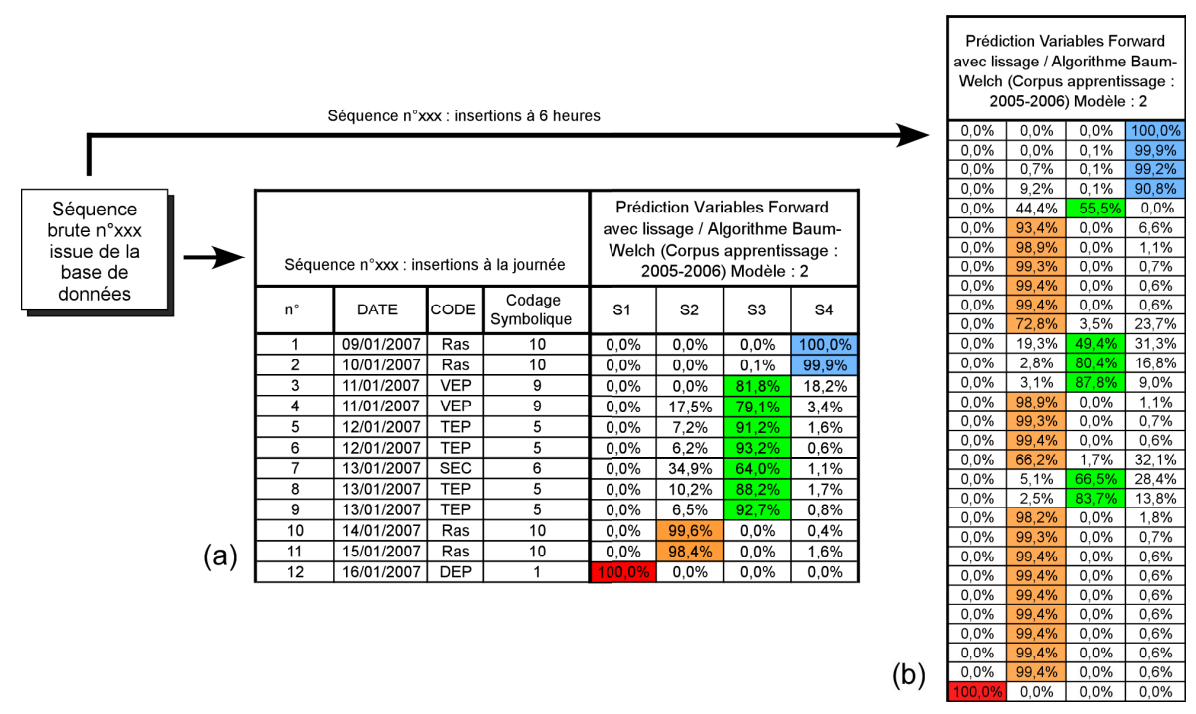

Figure 16. Résultats sur la séquence $n^{\circ} x x x$ à partir des deux types d'insertions : (a) : insertions à la journée, (b) : insertions à 6 heures

Dans le cas des résultats présentés (Figure 16 (a)), l'expert est alerté deux jours avant l'arrêt du système. Pour la Figure 16 (b), l'expert est alerté à la fois très souvent et bien trop en amont de l'arrêt effectif. Dans ces conditions, l'estimation 
prématurée de l'état $\mathrm{S} 2$ ne permet pas une planification optimale des opérations de maintenance car la "sur-insertion » d'observations apporte une trop grande sensibilité au modèle. Suite à une analyse globale concernant le critère d'insertions de symboles, nous avons retenu l'insertion journalière (situation qui correspond effectivement à l'organisation interne de la maintenance au sein de l'entreprise).

La Figure 17, montre pour une séquence, la concurrence que nous avions choisie d'adopter dans la qualité d'un modèle. Nous pouvons constater, dans ce cas, que la prédiction Viterbi avec lissage pour l'état S2 est bien trop rapide par rapport à une prédiction utilisant l'algorithme Variables Forward avec lissage.
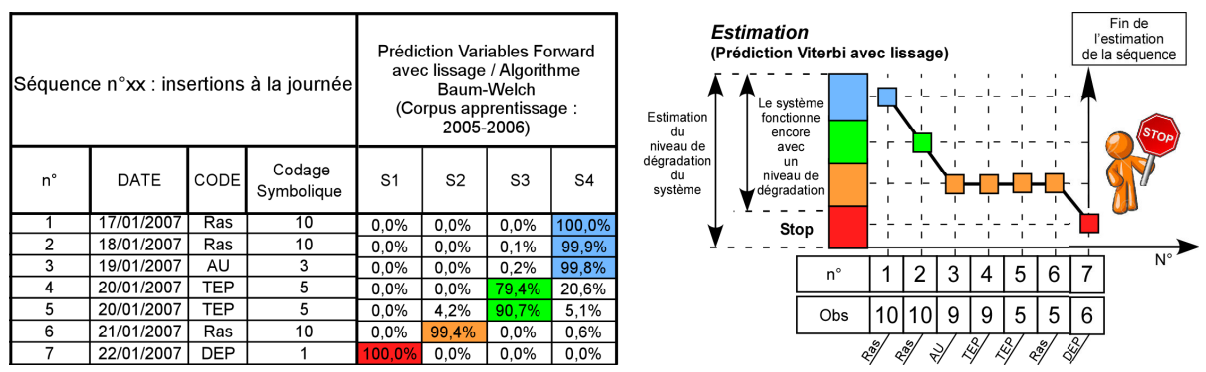

Figure 17. Résultats d'un test dépendant du modèle

Finalement, suite à des essais sur différentes séquences d'observations (corpus 2007), le modèle (prédiction Variables Forward avec lissage) a été retenu car il répond au mieux au critère de départ que nous nous étions fixés : "ne pas détecter la panne, ni trop tard, ni trop tôt ».

\subsubsection{Bilan des résultats : prédiction de pannes}

Le Tableau 10 propose une synthèse des résultats à la journée, concernant la capacité du modèle retenu à prédire l'arrivée de la panne. Ces résultats sont très encourageants. A +3 jours (si l'estimation perdure en S2), la probabilité de tomber en panne est de $85,7 \%$.

Si l'expert ne tenait compte que des statistiques, il pourrait se poser des questions sur l'arrivée possible de la panne à 5,38 jours (moyenne statistique sur 2005 et 2006 (Figure 9)). Cette information statistique n'étant par définition qu'un indicateur, elle ne peut à elle seule répondre aux exigences de l'expert.

\begin{tabular}{|c|c|c|c|c|c|c|}
\hline \multicolumn{7}{|c|}{ Probabilité d'être en panne sur le 1er trimestre 2007 } \\
\hline \multicolumn{6}{|c|}{$\begin{array}{c}\text { Prédiction Variables Forward avec lissage } \\
\text { (Apprentissage Baum-Welch) }\end{array}$} \\
\hline à + 1 jour & à + 2 jours & à + 3 jours & à + 4 jours & à + 5 jours & à + 6 jours & à + 7 jours \\
\hline $28,6 \%$ & $71,4 \%$ & $85,7 \%$ & $85,7 \%$ & $85,7 \%$ & $85,7 \%$ & $100,0 \%$ \\
\hline
\end{tabular}

Tableau 10. Prédiction de panne: Variables Forward avec lissage (peseuse M2) 


\subsection{Comparaison de l'approche MMC avec les lois de survie}

Le Tableau 11 permet, sur un exemple, de faire un point sur la capacité comparative des deux approches développées. L'expert disposant d'un tableau de ce type en temps réel pourrait avoir une vision globale sur le niveau de dégradation du système à partir de différents indicateurs. Au 14/01/2007, l'estimation du niveau de dégradation est dans l'état S2 (1ère alarme forte). Les autres indicateurs provenant des lois de survie estiment que le système a plus d'une chance sur deux d'être déjà tombé en panne.

\begin{tabular}{|c|c|c|c|c|c|c|c|c|c|c|c|}
\hline \multirow{2}{*}{\multicolumn{4}{|c|}{ Séquence $n^{\circ} 1$ : insertions à la journée }} & \multirow{2}{*}{\multicolumn{4}{|c|}{$\begin{array}{c}\text { Prédiction Variables Forward } \\
\text { avec lissage / } \\
\text { Algorithme Baum-Welch (Corpus } \\
\text { apprentissage : } \\
\text { 2005-2006) }\end{array}$}} & \multicolumn{4}{|c|}{ Modèles lois de survie } \\
\hline & & & & & & & & \multirow{2}{*}{\multicolumn{2}{|c|}{$\begin{array}{l}\text { Estimation } \\
\text { de } \\
\text { Kaplan-Meier } \\
\text { vec un intervalle } \\
\text { confiance de } 95 \%\end{array}$}} & \multirow{3}{*}{\begin{tabular}{|c|}
$\begin{array}{c}\text { Estimation } \\
\text { de } \\
\text { Kaplan-Meier }\end{array}$ \\
$4 \%$ \\
\end{tabular}} & \multirow{3}{*}{$\begin{array}{r}\operatorname{cox} \\
25 \%\end{array}$} \\
\hline \multirow{2}{*}{$\frac{\mathrm{n}^{\circ}}{1}$} & \multirow{2}{*}{\begin{tabular}{|c|} 
DATE \\
$09 / 01 / 2007$ \\
\end{tabular}} & \multirow{2}{*}{$\begin{array}{c}\text { CODE } \\
\text { Ras }\end{array}$} & \multirow{2}{*}{$\begin{array}{c}\begin{array}{c}\text { Codage } \\
\text { Symbolique }\end{array} \\
10\end{array}$} & \multirow{2}{*}{$\frac{s 1}{0,0 \%}$} & \multirow{2}{*}{\begin{tabular}{l|} 
S2 \\
$0,0 \%$
\end{tabular}} & \multirow{2}{*}{$\frac{\mathrm{s} 3}{0,0 \%}$} & \multirow{2}{*}{$\frac{\mathrm{S} 4}{100,0 \%}$} & & & & \\
\hline & & & & & & & & $1 \%$ & $8 \%$ & & \\
\hline 2 & $10 / 01 / 2007$ & Ras & 10 & $0,0 \%$ & $0,0 \%$ & $0,1 \%$ & $99,9 \%$ & $15 \%$ & $32 \%$ & $23 \%$ & $41 \%$ \\
\hline 3 & $11 / 01 / 2007$ & VEP & 9 & $0,0 \%$ & $0,0 \%$ & $81,8 \%$ & $18,2 \%$ & $27 \%$ & $45 \%$ & $36 \%$ & $54 \%$ \\
\hline 4 & $11 / 01 / 2007$ & VEP & 9 & $0,0 \%$ & $17,5 \%$ & $79,1 \%$ & $3,4 \%$ & $27 \%$ & $45 \%$ & $36 \%$ & $54 \%$ \\
\hline 5 & $12 / 01 / 2007$ & TEP & 5 & $0,0 \%$ & $7,2 \%$ & $91,2 \%$ & $1,6 \%$ & $38 \%$ & $57 \%$ & $47 \%$ & $66 \%$ \\
\hline 6 & $12 / 01 / 2007$ & TEP & 5 & $0,0 \%$ & $6,2 \%$ & $93,2 \%$ & $0,6 \%$ & $38 \%$ & $57 \%$ & $47 \%$ & $66 \%$ \\
\hline 7 & $13 / 01 / 2007$ & SEC & 6 & $0,0 \%$ & $34,9 \%$ & $64,0 \%$ & $1,1 \%$ & $48 \%$ & $66 \%$ & $57 \%$ & $73 \%$ \\
\hline 8 & $13 / 01 / 2007$ & TEP & 5 & $0,0 \%$ & $10,2 \%$ & $88,2 \%$ & $1,7 \%$ & $48 \%$ & $66 \%$ & $57 \%$ & $73 \%$ \\
\hline 9 & $13 / 01 / 2007$ & TEP & 5 & $0,0 \%$ & $6,5 \%$ & $92,7 \%$ & $0,8 \%$ & $48 \%$ & $66 \%$ & $57 \%$ & $73 \%$ \\
\hline 10 & $14 / 01 / 2007$ & Ras & 10 & $0,0 \%$ & $99,6 \%$ & $0,0 \%$ & $0,4 \%$ & $53 \%$ & $72 \%$ & $62 \%$ & $79 \%$ \\
\hline 11 & $15 / 01 / 2007$ & Ras & 10 & $0,0 \%$ & $98,4 \%$ & $0,0 \%$ & $1,6 \%$ & $59 \%$ & $76 \%$ & $67 \%$ & $87 \%$ \\
\hline 12 & $16 / 01 / 2007$ & DEP & 1 & $100.0^{\circ}$ & $0,0 \%$ & $0.0 \%$ & $0.0 \%$ & $68 \%$ & $84 \%$ & $76 \%$ & $93 \%$ \\
\hline
\end{tabular}

Tableau 11. Estimation du niveau de dégradation du système avec les deux approches (Peseuse M2)

Dans ces conditions, l'expert devrait réagir en fonction des différentes contraintes et priorités du terrain en considérant que s'il ne fait rien, il va vers la panne. Deux interventions de travaux préventifs ont été effectuées, suivies de deux journées sans aucune intervention et le processus tombe en panne.

La Figure 18 propose, pour la même séquence, une autre orientation dans la confrontation des deux approches. Les valeurs numériques des probabilités (Variables Forward) sont tirées du Tableau 10. Prenons le cas où l'expert fixerait les interventions de maintenance à partir d'une situation où le système aurait plus de $50 \%$ de chance d'être déjà tombé en panne. S'il se réfère uniquement aux lois de survie, il pourrait programmer une intervention (à - 4 jours (temps mesuré avant l'arrivée de la panne (le 16/01/2007), Figure 18)). S'il utilisait notre approche $\mathrm{MMC}$, il pourrait programmer une intervention à -1 jour. Cette différence de sensibilité peut s'expliquer par le fait que les lois de survie ne tiennent compte que du temps qui passe alors que notre modèle tient compte des observations collectées. Dans ce cas, notre modèle a bien identifié la « signature » d'une panne prochaine.

En considérant l'information S2, l'expert pourrait planifier des interventions de maintenance juste avant la panne. En effet, on peut supposer que la dégradation 
serait suffisamment marquée pour replanifier si la situation le permet, les actions de maintenance (Figure 4).

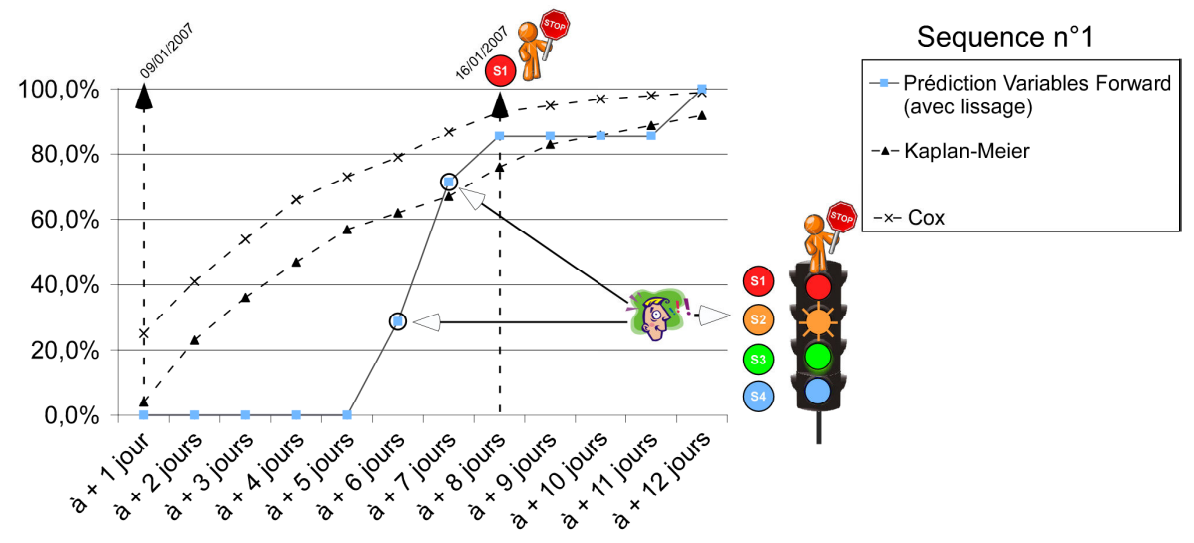

Figure 18. Probabilité de détecter la panne sur différentes simulations (peseuse M2)

\section{Conclusion et perspectives envisagées}

Notre approche montre que l'ensemble des résultats obtenus dans le cadre des deux études industrielles, peut apporter des éléments d'aide à la décision pour l'expert. Les résultats démontrent que notre système d'aide à la décision apporte une bonne estimation de la probabilité de tomber en panne lorsque l'on dispose d'observations. En l'absence d'évènements observables, les lois de survie pourraient prendre le relais. Après un travail mettant en relief les points forts ou faibles des différents algorithmes utilisés, nous avons mis en évidence que la procédure de simulations associée au préalable à un apprentissage du modèle $\lambda$, offrait les meilleurs résultats (apprentissage : Baum-Welch, prédiction : Variables Forward avec lissage). Nous avons aussi montré que le choix temporel concernant les insertions d'observations, avait un impact non négligeable sur la sensibilité de l'estimation. Pour l'étude de cas, nous voyons, qu'il est possible de faire cohabiter différents indicateurs (Figure 19) issus, soit des lois de survie utilisées, soit de notre approche MMC (Figure 18). 


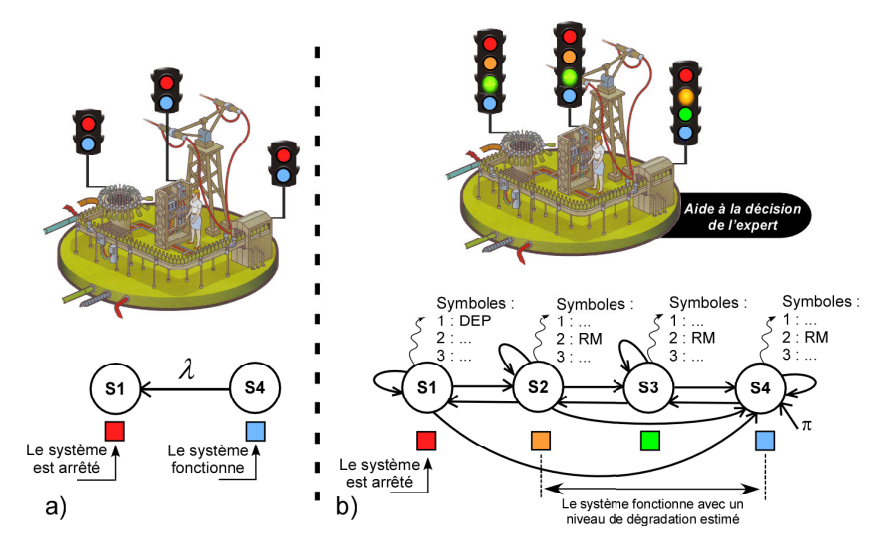

Figure 19. Kaplan-Meier et Cox (a) comparés à notre approche MMC (b)

De plus, nous montrons que l'expert disposant d'un tel outil d'analyse, peut tenter de qualifier les interventions de maintenance. Par exemple (Tableau 11), pourquoi le système tombe t-il en panne suite à deux interventions de travaux d'entretiens préventifs suivi de deux jours sans aucune intervention? Les premières analyses des évènements fournis par les industriels, leur ont permis de conforter leur connaissance des effets de certaines actions de maintenance. Les expérimentations ayant été effectués a posteriori, nous ne pouvons évaluer les effets de la programmation d'éventuelles actions de maintenance. Avant d'envisager la mise en place en situation réelle, nous allons explorer les possibilités de simulation d'un processus sur lequel nous appliquerions une planification dynamique de la maintenance.

Castanier et al., (2003), Dieulle et al., (2003) et Grall et al., (2002) proposent une gestion calendaire d'inspections. Cette gestion qui s'adapte à l'état de dégradation du système est construite de manière séquentielle. Les inspections sont d'autant plus fréquentes que le système est dégradé. Notre démarche de travail et les résultats que nous avons présentés issus de simulations dans le cadre de deux contextes industriels montrent qu'ils peuvent apporter des éléments complémentaires aux travaux exprimés par les différents auteurs cités dans cet article.

\section{Bibliographie}

AFNOR, Terminologie de la maintenance, NF EN 13306X 60-319, Juin, 2001.

Aupetit S., Contributions aux modèles de Markov cachés : Métaheuristiques d'apprentissage, nouveaux modèles et visualisation de dissimilarité, Thèse de doctorat, Université François-Rabelais, Tours, 2005. 
Avila M., Optimisation de modèles Markoviens pour la reconnaissance de l'écrit, Thèse de doctorat, Université de Rouen, 1996.

Bertholon H., Bousquet N. and Celeux G., "An alternative competing risk model to the Weibull distribution for modelling aging in lifetime data analysis", Lifetime Data Analysis, Volume 12, Number 4, 2006, pp. 481-504.

Basile O., Prise en compte de l'incertitude dans les modèles fiabilistes de maintenance industrielle. Extension aux sollicitations variables, Thèse de doctorat, Faculté Polytechnique de Mons, 2007.

Baum L.E., Petrie T., "Statistical Inference for Probabilistic Functions of Finite State Markov Chains", Annals of Math. Statistics, 37, 1966, pp. 1554-1563.

Baum L.E., "An inequality and associated maximization technique in statistical estimation for probabilistic functions of a Markov process ", Inequalities, 3, 1972, pp. 1-8.

Beltrami C., "e-maintenance - Possibilités actuelles et perspectives", PROCEED - A3SI Lorraine - ENSAM, 2005.

Birolini, A. Quality and reliability of technical systems, Edition Springer, 1994.

Breslow N. "Applications to clinical trials of multiple regression models for censored survival data", The Scientific Meeting of the Committee on Controlled Therapeutic Trials of the U.I.C.C, April, 1973.

Castanier B., Modélisation stochastique et optimisation de la maintenance conditionnelle des systèmes à dégradation graduelle Thèse de doctorat, Université de Technologie de Troyes, 2002.

Castanier B., Bérenguer C. and Grall A., "A sequential condition-based repair/replacement policy with non-periodic inspections for a system subject to continuous wear", Applied Stochastic Models in Business and Industry, 19(4), 2003, pp. 327-347.

Chandler A.D.Jr., Stratégie et structures de l'entreprise, Paris, Editions d'organisation, 1989.

Cheuk-Kit W., Eng Wie T. "Survival over 5 years in the initial hospital survivors with acute coronary syndrome: a comparison between a community hospital and a tertiary hospital in New Zealand", Journal of the New Zealand Medical Association, vol. 120, $\mathrm{n}^{\circ} 1261$, 2007.

Cox D.R. "Regression Models and Life Tables (with Discussion)", Journal of the Royal Statistical Society, Series B 34:187-220, 1972.

Despujols A., « Approche fonctionnelle de la maintenance », Techniques de l'ingénieur, MT $9020,2004$.

Dieulle L., Bérenguer C., Grall A. and Roussignol M., "Sequential condition based maintenance scheduling for a deteriorating system", European Journal of Operational Research, 150, 2003, pp. 451-461.

Gouriéroux C. Econométrie des variables qualitatives, Paris, Economica, 2ème édition, 1989.

Grall A., Bérenguer C. and Dieulle L., A "condition-based maintenance policy for stochastically deteriorating systems", Reliability Engineering and System Safety, 76, 2002, pp. 167-180. 
Grundy W.N., Bailey T.L., Elkan C.P., Baker M.E., "Meta-MEME : Motif-based Hidden Markov Models of protein families", Computer applications in the biosciences, 13(4), 1997, pp. 397-406.

Hastings N.A.J, "The repair limit replacement method", Operational Research Quarterly, 20, 1969, pp. 337-349.

Kalbfleisch J.D., Prentice R. L., The Statistical Analysis of Failure Time Data, 2nd edition, John Wiley \& Sons, New York, 2002.

Leger J.B., "Maintenance \& Transports : De la e-maintenance de sites industriels... à la emaintenance de flottes", PREDICT, 2007.

Meier-Hirmer C., Pouligny P., Ardeois J., Sibille N., « Optimisation de l'utilisation des trains meuleurs pour la maintenance des voies ferrées ", Maîtrise des risques et sûreté de fonctionnement, Im16, 4A-3, 2008.

Ministère de l'Écologie de l'Energie du Développement durable et de la Mer, Renforcement de la politique de prévention des risques liés aux inondations, 2006.

Ministère de l'intérieur, Présentation du nouveau plan gouvernemental de vigilance, de prévention et de protection face aux menaces d'actions terroristes : Vigipirate, premierministre.gouv.fr, 2003.

Monchy F., La fonction maintenance : Formation à la gestion de la maintenance industrielle Collection technologies de l'université à l'industrie, MASSON, 1991.

Muller A., Contribution à la maintenance prévisionnelle des systèmes de production par la formalisation d'un processus, Thèse de doctorat, Université de Nancy I, 2005.

Muller A., Crespo Marquez A. and Iung B., "On the concept of e-maintenance: Review and current research", Reliability Engeniering and System Safety 93, 2008 pp. 1165-1187.

Muth E.J., "An optimal decision rule for repair versus replacement", IEEE Transactions on Reliability, R-26, 1997, pp. 179-181.

Pacaut P., Le Bourdais C., Laplante B. «Dynamique et déterminants de la participation des femmes au marché du travail après la naissance d'un enfant au Canada» Cahiers québécois de démographie, Volume 36, numéro 2, 2007, pp. 249-279.

Phelps R.I., "Replacement policies under minimal repair", Journal of the Operational Research Society, 32, 1981, pp. 549-554.

Rabiner L.R., "A tutorial on hidden Markov models and selected applications in speech recognition", Proceeding of the IEEE, vol. 77, 1989, pp. 257 - 286.

Schalapbach A., Bunke H., "Using HMM-based recognizers for writer identification and verification", Workshop on frontiers in Handwriting Recognition, Proceeding. $9^{\text {th }}$ International, 2004, pp. 167 - 172.

Signoret J.P., «Analyse des risques des systèmes dynamiques : approche markovienne», Techniques de l'ingénieur, SE 4 071, 2005.

Simeu-Abazi Z. and Sassine C., "Maintenance integration in manufacturing systems by using stochastic Petri nets", International Journal of Production Research, 37, 1999, pp. 39273940. 
Soro I-W., Nourelfath M. and Aït-Kadi D., "Performance Evaluation of multi-state degraded systems with minimal repairs and imperfect maintenance", Reliability Engineering and System Safety, 2010, pp. 65-69.

Tarondeau J.C. , Lorino P., «De la stratégie aux processus stratégiques », Revue Française de la gestion, $\mathrm{n}^{\circ} 117,1998$, pp. 5-17.

Thiétart R.A., Martinet A.C., Posture paradigmatique et recherche en management stratégique, Paris, Stratégies : actualités et futurs de la recherche, Vuibert, 2001.

Thomas E., Levrat E., Iung B. and Monnin M., “Odds algorithm'-based opportunity triggered preventive maintenance with production policy”, 6th IFAC Symposium Safeprocess'06, China, 2006, pp. 835 - 840.

Valdez-Florez C. and Feldman R.M., "A survey of preventive maintenance models for stochastically deteriorating single-unit systems", Naval Research Logistics, 36, 1989, pp. 419-446.

Villemeur A. Sûreté de fonctionnement des systèmes industriels : Fiabilité, Facteurs humains et Informatisation, Collection EdF - Ed Eyrolles, 1988.

Viterbi A.J., "Error bounds for convolutional codes and asymptotically optimum decoding algorithm”, IEEE Trans. on Information Theory, 13 (1967), pp. 260 - 269.

Wang H., "A survey of maintenance policies of deteriorating systems", European Journal of Operational Research, 2002, pp. 469-489.

Welte T., Deterioration and Maintenance Models for Components in Hydropower Plants, Ph D thesis, Norwegian University of Science and Technology, Department of Productions and Quality Engineering, 2008.

www.fr.wikipedia.org/wiki/Maintenance

Zille V., Bérenguer C., Grall A., Despujols A., Lonchampt J., "Modelling and performance assessment of complex maintenance programs for multi-component systems", In ESREDA $32^{\text {nd }}$ Seminar proceeding, Alghero, 2007. 